\title{
Dictynna
}

Dictynna

Revue de poétique latine

$7 \mid 2010$

Varia

\section{Corolles pour Narcisse}

Une lecture ovidienne au cœur d'un centon virgilien

\section{Gilles Tronchet}

\section{OpenEdition}

Journals

Édition électronique

URL : http://journals.openedition.org/dictynna/354

DOI : 10.4000/dictynna.354

ISSN : 1765-3142

Référence électronique

Gilles Tronchet, «Corolles pour Narcisse », Dictynna [En ligne], 7 | 2010, mis en ligne le 23 novembre 2010, consulté le 10 septembre 2020. URL : http://journals.openedition.org/dictynna/354; DOI :

https://doi.org/10.4000/dictynna.354

Ce document a été généré automatiquement le 10 septembre 2020.

\section{(c) (†) $\odot$}

Les contenus des la revue Dictynna sont mis à disposition selon les termes de la Licence Creative Commons Attribution - Pas d'Utilisation Commerciale - Pas de Modification 4.0 International. 


\title{
Corolles pour Narcisse
}

\author{
Une lecture ovidienne au cœur d'un centon virgilien ${ }^{1}$
}

\section{Gilles Tronchet}

1 Forme de poésie régulièrement pratiquée entre les deuxième et sixième siècles de notre ère, le centon antique offre la particularité de se construire exclusivement grâce à de courts fragments prélevés ici ou là dans les œuvres d'un même auteur, essentiellement Homère pour les Grecs et Virgile pour les Latins, de manière à composer un poème inédit. Ce genre de poème diffère donc de l'approche moderne la plus répandue, où il est constitué d'extraits, en général des vers, issus d'une série d'œuvres a priori illimitée ${ }^{2}$. La stricte détermination du réservoir où l'on peut puiser donne une plus grande évidence au rapport d'engendrement: le produit tend à offrir une impression de familiarité, sans que l'on ait besoin d'identifier le passage exact d'où provient chaque vers.

Quand on la confronte avec la démarche des autres poètes antiques, la procédure suivie par les centonistes, même si elle détermine un type d'écriture inhabituel, manifeste un solide ancrage dans la tradition. En effet, reprendre comme un matériau de base les poèmes de ses prédécesseurs, imiter leurs vers tout en modifiant les contextes, correspondait à une pratique très répandue, au moins depuis l'époque hellénistique, et qui a eu tendance à devenir pour la poésie latine une norme implicite.

De plus, la récriture savamment concertée de compositions antérieures, telle que la pratiquaient presque tous les écrivains, mobilisait très largement des modèles canoniques, des œuvres dont la place qu'elles avaient reçue dans l'éducation avait fait les supports d'une mémoire collective, les monumenta de la société cultivée: il en allait ainsi d'abord pour les poèmes homériques, et par la suite, dès le premier siècle de notre ère, pour les œuvres de Virgile ${ }^{3}$.

4 Ovide se place en quelque sorte au carrefour de ces approches: très apprécié dès son époque, il se montre lui-même un assidu lecteur et un adaptateur précoce de Virgile, dont les œuvres ont exercé une forte influence, directe ou indirecte, sur ses productions. En particulier les Métamorphoses font écho à de nombreux hexamètres virgiliens, tandis que plusieurs épisodes reprennent et développent certaines situations narratives, issues notamment de l'Enéide ${ }^{4}$. Mais Ovide a bénéficié non moins d'une 
notoriété durable, et il a été beaucoup lu au cours des siècles suivants, comme en témoignent de fréquents échos chez les poètes, depuis les tragédies de Sénèque, en passant par Stace ou Martial, jusqu'à l'époque de Claudien, à la fin du quatrième siècle: il suffit de parcourir l'œuvre la plus célèbre de cet écrivain, Le Rapt de Proserpine, pour y déceler comment, plus que toute autre, l'influence d'Ovide se conjugue à celle de Virgile ${ }^{5}$.

5 Cette renommée a pu déboucher sur la composition de centons ovidiens, dont on ne possède il est vrai que l'indice ${ }^{6}$. Mais elle se traduit de manière plus concrète par l'existence de centons virgiliens qui empruntent aux Métamorphoses leur sujet. Ces pièces font partie d'un recueil réalisé en Afrique au sixième siècle et inclus par la suite dans l'Anthologie Latine'. L'une reprend l'enlèvement d'Europe par Jupiter déguisé en taureau; une autre est dédiée à Narcisse, lorsque, dans un décor naturel séduisant, il tombe amoureux de sa propre image réfléchie par les eaux et demeure captif de ce reflet $^{8}$.

6 C'est ce bref poème de 16 vers que j'ai choisi d'étudier, à cause du relief particulier qu'y prend, me semble-t-il, la lecture du récit ovidien: on la discerne à travers les similitudes que le centoniste parvient à ménager avec ce modèle, mais aussi dans les transformations qu'il lui apporte. L'un de ses aspects les plus marquants est d'offrir par endroits, grâce à des formules empruntées à Virgile, une physionomie qui n'est pas sans évoquer certains énoncés de l'épisode offert dans les Métamorphoses ${ }^{9}$ : un choix subtil des fragments virgiliens a permis d'intégrer des ressemblances notables avec le phrasé ovidien, qu'elles se fondent sur des échos reliant les deux œuvres préexistantes ou sur un montage inventif autorisant les coïncidences inédites ${ }^{10}$.

7 On observe ainsi un maniement complexe et maitrisé de la double référence qui régit l'écriture, dépendante à la fois du corpus virgilien, comme fournisseur exclusif de ses formulations (on peut parler à cet égard d'œuvre-source), et de l'épisode ovidien, comme déterminant global de la diégèse (on peut parler à cet égard d'œuvre-cadre) ${ }^{11}$. Une interaction s'établit entre les correspondances reliant le centon à chacune des deux œuvres: elle atteste une confrontation soigneuse de celles-ci, qui a permis d'identifier les points de rencontre qu'elles offraient pour en tirer parti. Sans nul doute le sujet retenu, fondé sur le motif du reflet, qui fait vaciller la distinction entre l'unique et le double, s'harmonise à merveille avec une composition qui, en diversifiant les réminiscences, figure à plus d'un titre comme un miroitement de textes antérieurs.

Tout d'abord, j'observerai brièvement la manière dont s'agence le centon de Narcisse, en regard des principes avancés par Ausone dans la lettre au rhéteur Paulus qu'il a jointe à son Centon nuptial. Ensuite, j'analyserai les rapports entre l'œuvre anonyme et le récit des Métamorphoses, qu'elle reprend dans ses grandes lignes, pour ainsi dire en abrégé, non sans quelque difficulté parfois pour le lecteur, quand l'assemblage des fragments résiste à l'interprétation. Enfin, j'étudierai comment plusieurs aspects typiques du texte ovidien se trouvent habilement réinvestis dans la trame virgilienne. Je souhaite faire ainsi ressortir l'inventivité de la démarche suivie par le centoniste qui parvient à transposer sur un autre plan les données de l'œuvre-cadre ${ }^{12}$.

\section{L'actif d'un florilège: découverte d'un écrit insolite}

9 Dans sa lettre à Paulus, Ausone, on le sait, privilégie une découpe qui scinde les vers de l'original, Virgile en l'occurrence, pour empêcher que les hexamètres, avec leur unité 
de rythme, ne soient immédiatement reconnaissables et ne reportent le lecteur au modèle, plutôt que de tendre à se fondre dans le nouveau contexte. C'est pourquoi il oppose un net refus aux séquences trop massives, parce qu'elles convoqueraient directement la mémoire du passage impliqué dans l'œuvre-source ${ }^{13}$. Selon cette logique de rupture avec les ensembles prédéterminés dans l'œuvre-source, il préconise des modules d'un demi-vers ou d'un vers et demi, en s'abstenant de mentionner l'emploi d'un unique vers entier, même s'il y recourt assez fréquemment ${ }^{14}$. Du reste, les extraits monostichiques, dont le rôle est majeur dans les centons grecs dérivés d'Homère, reçoivent dans la production latine une place qui n'est pas négligeable ${ }^{15}$. Un simple coup d'œil sur le centon de Narcisse, avec l'indication des extraits virgiliens qu'il intègre ne révèle aucun groupement de vers issus de l'œuvre-source, dont la présence aurait pesé d'un poids excessif dans un ensemble aussi restreint ${ }^{16}$; en revanche, on repère trois hexamètres complets sur seize (v. 7, 9 et 12), le reste étant formé de vers fragmentés ${ }^{17}$.

10 L'évidente propension du poète à scinder les vers mobilisés chez Virgile est conforme à l'esprit des instructions que formule Ausone. Celui-ci prend d'ailleurs soin d'expliquer les diverses manières dont le découpage peut s'effectuer: il préconise le respect des césures habituellement pratiquées ${ }^{18}$. À cet égard, dans le centon anonyme, le v. 6 désobéit à la règle, puisque ses deux composantes ne coïncident pas avec des hémistiches rythmiques ${ }^{19}$. Mais ce cas reste isolé20.

11 En fait, les préceptes d'Ausone, même s'ils n'ont qu'une valeur indicative, correspondent à des orientations générales qui dominaient la production centoniste. Pourtant, ils devaient s'appliquer avec une rigueur variable selon les écrivains et les difficultés rencontrées ${ }^{21}$. En tout état de cause, pour Ausone lui-même, c'est l'effet d'ensemble qui importe, davantage que le respect scrupuleux des règles: il ne faut pas qu'un montage mal ajusté fasse ressortir l'indépendance originelle des éléments employés. Cela risque d'advenir quand des raccourcis provoquent un encombrement syntaxique gênant pour la compréhension du passage, ou qu'au contraire la difficulté pour articuler directement les extraits suscite, dans l'intervalle, le recours à une transition superfétatoire, une sorte de cheville ${ }^{22}$.

Du coup, dans ses recommandations, Ausone insiste sur la nécessité de franchir l'étape d'un simple assemblage (iunctura) pour aboutir à une cohérence harmonieuse (concinnatio) ${ }^{23}$. Cela traduit une indéniable conscience de la pression qui s'exerce sur l'écriture du centon, des problèmes qui se posent pour obtenir malgré tout une représentation satisfaisante: il s'agit d'éviter les gaucheries dues à la contrainte et produisant l'ellipse, l'anacoluthe ou la surcharge.

Quand on le parcourt en entier, le centon de Narcisse offre un agencement assez homogène, où ressortent malgré tout plusieurs bizarreries sémantiques. On remarque dès la première phrase l'emploi quelque peu abrupt d'un sujet abstrait, iuuentus, « la jeunesse », pour iuuenis, « un jeune homme ${ }^{24}$ ». Mais c'est surtout le v. 10 qui résiste un moment à l'élucidation: l'ablatif de lieu, dorso... iniquo, chez Virgile, évoque une aspérité du fond sur lequel un bateau s'est échoué; il doit s'interpréter ici comme une formule désignant le bombement irrégulier de la berge où Narcisse demeure en arrêt ${ }^{25}$.

De fait, on découvre ensuite que le jeune homme contemple son image dans des eaux en mouvement (fluminis undas, v.13), au lieu du miroir immobile qu'offrait la source limpide évoquée chez Ovide (M. III, 407-412), détail repris d'ailleurs au début du centon (v. 3). Il est vraisemblable que ce léger écart atteste le tribut que la composition a dû 
payer au corpus virgilien, comme ensemble clos de données limitant l'éventail des formules disponibles: à cet endroit du poème, le centoniste a privilégié la notation d'un lieu isolé (secreti) fournie par un extrait apte à s'incorporer dans le contexte immédiat, quitte à introduire un menu décalage par rapport aux indications précédentes, fidèles à la description de l'œuvre-cadre.

La pression qui s'exerce de cette manière sur les trouvailles possibles implique une discipline d'écriture préalable: la teneur globale du propos doit se guider sur un échantillon d'occurrences effectives, de façon à déterminer un programme exécutable qui impose à son tour ses exigences au choix précis des extraits, afin de réussir l'ajustement de détail. De la sorte, une double adaptation s'avère indispensable pour faire correspondre le réservoir, assez vaste au demeurant, avec le développement des motifs envisagés par le centoniste ${ }^{26}$.

Une interaction doit s'établir entre le scénario, dont les particularités vont se préciser peu à peu, et les fragments susceptibles d'être mobilisés: il est probable que l'élaboration s'effectuait par tâtonnements, de manière à découvrir les biais qui permettraient de surmonter les obstacles, tantôt en pliant la représentation aux exigences du matériau mobilisable, tantôt en aménageant celui-ci. En ce cas, une découpe adéquate est susceptible de transformer la fonction d'un fragment ou d'affecter à certains mots une signification toute différente ${ }^{27}$. Comme le souligne Françoise Desbordes, l'unité requise par le centon est le syntagme, dans le cadre d'un vers ou plus souvent d'un hémistiche, et elle ne prend sa valeur que par rapport à un alentour qui l'intègre, mais dont elle conditionne en même temps la physionomie ${ }^{28}$.

Or la complexité de la démarche s'accroît avec la référence à une œuvre-cadre, guidant l'anecdote et contraignant le centoniste à respecter dans ses grandes lignes un déroulement préétabli: il en va ainsi pour le centon de Narcisse, où le redoublement des modèles prend une tournure d'autant plus intéressante qu'il a conduit à ménager toute une série d'échos rappelant de façon précise le récit ovidien. On observe en quelque sorte un ensemble de réminiscences couplées, parce que le centoniste a su prélever dans le corpus virgilien des fragments susceptibles, selon un paradoxe temporel, de rappeler certains passages d'un poète... ultérieur, Ovide en l'occurrence ${ }^{29}$. Avant d'examiner ce curieux phénomène de double filiation, il importe de confronter le florilège qu'offre le centon avec l'épisode contenu dans les Métamorphoses: on va voir comment se dégage une lecture qui a su déceler dans l'œuvre-cadre certaines lignes de force que la composition est parvenue ensuite à restituer comme en raccourci.

\section{L'agencement du bouquet: dérivation d'un modèle narratif}

Un survol du centon anonyme consacré à Narcisse donne l'immédiate impression d'un abrègement extrême par rapport à la version d'Ovide: 16 hexamètres au lieu de 172 ( $M$. III, 339-510 ${ }^{30}$. Il est vrai que l'histoire ne reprend pas le développement consacré à la vaine passion d'Écho pour le bel adolescent (v. 356-401). Mais on comprend sans mal que, dans sa brièveté, le poème se focalise sur le héros, en omettant la réduplication interne qu'introduisaient la nymphe et sa métamorphose, avec la mise en scène de répliques vocales, répondant au motif du miroitement ${ }^{31}$. Car l'aventure du seul Narcisse illustre suffisamment par elle-même le thème du double. C'est pourquoi le centon, dont 
la facture incarne justement le retour partiel d'énoncés, n'exigeait pas la mise en scène de l'écho ${ }^{32}$.

19 En fait, malgré sa différence d'échelle et l'importance de ses décalages avec le scénario imaginé par Ovide, le centon possède suffisamment de traits communs avec le récit des Métamorphoses pour que l'on puisse attribuer à celui-ci le statut d'œuvre-cadre. On observe le développement d'une récriture concertée, qui n'est pas fondée sur un décalque diégétique mais sur une réinterprétation des éléments narratifs. Une telle démarche suppose une lecture précise, capable de saisir dans l'épisode ovidien certains aspects décisifs dont la composition, jouant sur les possibilités qu'offre son matériau de base, s'attache à fournir des équivalents. Une indéniable corrélation d'ensemble se dégage, quand on observe par étapes de quelle façon les éléments du récit ont été repris, infléchis ou éludés ${ }^{33}$.

\section{La présentation du héros}

20 Elle s'inspire directement des Métamorphoses, signalant d'emblée qu'il s'agit là d'un modèle narratif: dès le début (v. 1) est évoquée la jeunesse de Narcisse, ainsi que son extraordinaire beauté, sur laquelle revient avec insistance le v. 4 . Le choix de l'adjectif candida pour amorcer le poème correspond à l'accent mis par Ovide sur la blancheur de neige qui distingue le teint du jeune homme (M. III, 423 et 491$)^{34}$. La bizarrerie de la formulation réside, on l'a vu, dans le recours à l'abstrait iuuentus, « la jeunesse », pour désigner le jeune homme (iuuenis chez Ovide, v. 352) ${ }^{35}$. Cependant, le terme usuel apparaît plus loin (iuuenem, v. 8), à l'instant où Narcisse découvre son image et croit voir un autre adolescent ${ }^{36}$. La mention renouvelée de sa beauté rappelle alors, en abrégé, l'évocation des Métamorphoses (v. 416-423). Différée jusque-là, une précision à propos de sa naissance complète le vers: le héros est le fils d'une nymphe, comme Ovide l'indiquait dès le départ (v. 342-346) ${ }^{37}$.

21 À cet endroit, le centon présente une anomalie: dans la phrase, le détail fourni se rapporte à l'inconnu contemplé par Narcisse. Or, le récit observe jusque-là un régime de focalisation interne centré sur le héros qui tombe amoureux d'un jeune homme, sans savoir, justement, qu'il est confronté à sa propre image. Comment Narcisse pourrait-il être informé sur les origines de cet inconnu sans consistance? Tout se passe donc comme si le narrateur intervenait soudain, anticipant la mise en équivalence des deux personnages, selon une forme d'intrusion, que l'on pourrait nommer, en transposant la terminologie de Gérard Genette, une métalepse de focalisation ${ }^{38}$.

22 Cette approche, neutralisant la voix du narrateur hétérodiégétique, si présente dans les Métamorphoses, tranche par rapport à l'insistance du récit ovidien sur la réduplication, traduite par de nombreux réfléchis et un jeu réunissant l'actif et le passif ${ }^{39}$. Pourtant, l'ambiguïté du rapport à soi comme à un autre inaccessible est bien mise en valeur grâce à la périphrase qui objective Narcisse à travers son double. Aussi la maladresse n'est-elle qu'apparente: l'étrangeté représentative à la fin du v. 8 , juste à la charnière centrale du poème, préfigure sur un mode allusif le basculement qui s'opère dans la phrase suivante, où le narrateur, désormais en position de surplomb, explique la situation, tout en marquant l'ignorance du héros.

Quant à l'insensibilité hautaine du Narcisse ovidien repoussant les avances de celles et ceux qui sont amoureux de lui, la fin du v. 5 en offre un discret indice: le héros, lorsqu'il parcourt la forêt et contemple ses beautés, ne redoute pas de succomber à la passion ${ }^{40}$. 
Par rapport au précédent hexamètre, la similitude qu'établit le retour du même mot terminal (amorum/amorem) accentue un contraste révélateur: même s'il ne craint rien, le jeune homme apparaît d'avance comme perméable à des sentiments que le charme du lieu distille en son cœur ${ }^{41}$.

\section{L'évocation du décor} schématique de la description offerte dans le récit ovidien, juste avant l'instant décisif où Narcisse va être puni de ses dédains ${ }^{42}$ : une forêt (v. 1) qui abrite une source sacrée (v. 3) rappelle les frais ombrages des Métamorphoses (III, 412) ainsi que la fontaine dont jamais la surface n'a été troublée (v. 407-410). Au tableau manque un seul détail, le moelleux tapis de gazon où le héros peut venir s'étendre (M. III, 411) ${ }^{43}$. Il est vrai que la fin du v. 2 mentionne les "présents du ciel ", caelestia dona, les divins agréments qui caractérisent l'endroit ${ }^{44}$. La formule, énigmatique au premier abord, semble capable de suggérer, dans le contexte, les délices d'une prairie émaillée de fleurs ${ }^{45}$. En effet, elle est liée par une conjonction de coordination à la présence de l'eau, détail qui, de pair avec l'ombre des arbres, donne pleine consistance à la caractérisation emphatique du paysage que célèbre le jeune homme. L'ensemble suggère ainsi, avec une indéniable économie de moyens, l'image d'un site analogue à celui où Narcisse, dans le récit d'Ovide, est pris au piège.

Ce genre d'endroit, on le sait, sous les dehors les plus accueillants, se révèle très souvent redoutable, dès lors qu'il s'inscrit dans un contexte mythique. Au début du centon, l'atmosphère solennelle qu'installent les adjectifs caelestia et sacros retrouve indirectement l'ambiance propre à l'épisode ovidien. Elle marque la présence de divinités tutélaires, dont un acte innocent risque d'éveiller le ressentiment. Chez Ovide, la mise en place du décor fait suite aux malédictions d'un amoureux éconduit (v. 404-405): elles exercent leur effet quand le héros s'abreuve à la source, comme s'il portait atteinte à un lieu sacré, jusqu'alors inviolée ${ }^{46}$.

Mais si le centoniste offre l'ébauche d'un site comparable à celui des Métamorphoses, il renonce d'emblée à calquer la diégèse sur celle de l'œuvre-cadre. En effet, dès la première phrase, le recours à l'imparfait transforme l'allure globale du récit et détermine le caractère itératif de l'action. Au lieu de découvrir une fois pour toutes l'asile privilégié dont il chante les louanges, Narcisse y multiplie les visites, au cours desquelles il s'abreuve d'un amour encore vague et diffus.

Cela retarde l'événement crucial, le surgissement de l'amour pour soi-même, qui coïncide chez Ovide avec l'arrivée près de la source. Cependant, la communauté entre le centon et l'œuvre-cadre est maintenue par le biais d'une métaphore, l'emploi de bibebat au v. 4: Narcisse "buvait» la passion en contemplant le paysage, peu à peu gagné par un état d'âme qu'insuffle le lieu, sans que soit encore impliqué son reflet. En retenant ainsi une image que Virgile utilisait à propos de Didon (En. I, 749), le centoniste adapte un jeu ovidien entre sens propre et figuré: il s'agissait d'un polyptote sur le nom sitis, «la soif » (v. 415) ${ }^{47}$.

Ovide soulignait une rencontre paradoxale entre le désir insatiable et le simple souci de se désaltérer. La récriture se fonde sur une inversion de la métaphore: l'effet de l'amour n'est plus d'assoiffer, mais d'imprégner l'être. La recherche dans l'œuvre-source d'un 
équivalent pour le geste de Narcisse buvant à la fontaine (bibit, v. 416) a suscité un choix qui, grâce à un habile détournement, autorise l'emploi du même verbe ${ }^{48}$.

Il semble que cette double correspondance ait guidé le centoniste, le conduisant à employer l'imparfait, conformément à l'extrait disponible, alors qu'il n'était pas démuni d'une solution au présent ${ }^{49}$. Ainsi, la différence des temps par rapport à l'œuvre-cadre, notable surtout au début du centon, pourrait ne pas être un simple biais représentatif, imposé par les contraintes de l'exercice, mais le produit d'un arbitrage entre les options du scénario et les données utilisables.

Un tel exemple signale la relative marge de manœuvre dont bénéficie la composition centonale, pour prélever les fragments et les articuler. Son effort ne se borne pas forcément à décalquer une histoire, en détectant les solutions loisibles dans le corpus. C'est pourquoi, les écarts par rapport au modèle, loin de se ramener forcément à des altérations provoquées par l'obéissance aux lois du genre, manifestent parfois une logique autonome, arbitrant les rapports avec l'œuvre-source et l'œuvre-cadre.

On ne saurait analyser de la même manière la présence dans le paysage d'un élément inédit, le cours d'eau près duquel Narcisse demeure fasciné par son image (v. 13). Ce détail n'est pas tout à fait conforme au scénario des Métamorphoses, où le jeune homme restait prostré auprès d'une nappe d'eau formée par la source ${ }^{50}$. En outre, comme il provient d'un extrait virgilien où flumen désigne bel et bien une rivière, il donne l'impression d'un brusque changement de cadre, qui tend à en faire vaciller quelque peu la cohérence ${ }^{51}$. Certes, il est possible d'établir une connexion entre source et cours d'eau, mais elle réside, pour ainsi dire, dans les marges du poème, un passage de Virgile dont le centon n'intègre qu'une partie: à l'origine, le fragment qui mentionne la source, dont on a vu l'importance pour restituer le décor ovidien, est couplé avec l'évocation de flumina, pour évoquer un paysage riant (B. I, 51-52) ${ }^{52}$.

Ainsi, la venue d'un cours d'eau peut trouver dans l'œuvre-source une motivation, mais celle-ci, exogène aux vers du centon, ne semble pas suffisante pour amortir une certaine rudesse dans l'enchaînement des notations. Cette analyse confirme le diagnostic avancé plus haut ${ }^{53}$ : même si la démarche se fonde sur une analogie, c'est faute de mieux que la fin du v. 13, sans qu'un raccord narratif clair vienne le justifier, modifie le site de la contemplation narcissique.

Quoi qu'il en soit, la lecture qu'accomplit le centoniste en récrivant l'épisode des Métamorphoses offre l'intérêt d'attribuer d'abord au paysage la séduction qui opère sur Narcisse. Ainsi, la pulsion scopique, si prégnante dans le texte d'Ovide, s'affirme nettement à propos du locus amoenus, dont le caractère érotique demeurait implicite dans les Métamorphoses ${ }^{54}$ : les regards pénétrants que lance le jeune homme (v. 5) s'attardent sur les charmes du décor, avant de le conduire jusqu'à sa propre image. Aussi les lieux tendent-ils à être présentés comme un équivalent de Narcisse lui-même: la reprise de l'adjectif insignis, aux v. 3 et 4, assimile la beauté du personnage avec la célébration du site $\mathrm{s}^{55}$. Tout est donc prêt pour que l'engouement de Narcisse envers un décor dans lequel il est voué à se fondre soit converti en amours réflexives. C'est sur cette base que le centoniste réaménage le scénario d'Ovide.

\section{La construction du récit}

L'enjeu global de l'intrigue, le piège réfléchissant dont est victime le héros, n'est dévoilé qu'à mesure dans le centon, selon une stratégie qui retarde la compréhension 
d'ensemble ${ }^{56}$. Ce dispositif, même s'il n'appartient pas à l'œuvre-cadre, est en adéquation avec l'intrigue commune, fondée sur la méprise. La problématisation de l'identité à travers le clivage dû au reflet, comme la réalisait exemplairement le récit ovidien, trouve un intéressant prolongement dans l'emploi d'un registre ambigu, révélant à mesure l'identité de Narcisse avec cet autre qu'il contemple: celui-ci, dans le v. 9, s'avère d'abord d'une ressemblance parfaite ${ }^{57}$; puis, dans le v. 10, l'unicité s'affirme, non sans ambivalence, avec la formulation, his amor unus erat, "un seul amour les unissait », renvoyant dans l'Enéide à l'union de Nisus et Euryale. C'est l'occasion pour le centoniste de faire discrètement écho à la formule qu'employait le Narcisse ovidien, apostrophant son image, puer unice (" enfant sans pareil», M. III, $454)^{58}$. Cependant, aux v. 11-12, le personnage n'arrive pas à prendre vraiment conscience du redoublement qui s'opère: il appartient au seul récit de marquer une identité qui ne devient manifeste qu'avec le premier hémistiche du v. 14.

Dans son ensemble, l'histoire se développe en trois temps: une attirance dépourvue d'objet s'insinue dans le cœur du héros sans qu'il y prenne garde, sous l'influence du décor (v. 1-5); puis il s'éprend d'un beau jeune homme, des formules ambigües laissant croire à la présence d'un second personnage, très ressemblant (v. 6-9); enfin le narrateur indique l'erreur de Narcisse qui, néanmoins, reste asservi à son désir pour un simple reflet (v. 10-16).

Cette disposition ternaire est conforme à la structure narrative de l'œuvre-cadre, malgré de notables disparités dans la diégèse. En effet, le récit ovidien comporte clairement trois étapes: les résistances de Narcisse à l'amour, notamment à celui d'Écho (v. 351-406), puis la passion qui le saisit pour un adolescent dont il ignore qu'il se confond avec lui (v. 407-462), enfin, quand il comprend qu'il s'agit de son double, l'enlisement fatal dans une situation qui n'a d'autre issue que sa mort, débouchant sur une métamorphose (v. 463-510).

De plus, si l'on restreint l'épisode à la mésaventure du héros, il est facile d'isoler à nouveau un triptyque: la découverte de la source (v. 407-436), les paroles que prononce Narcisse (v. 437-473) et la consomption qui aboutit à sa transformation en fleur (v. 474-510). Cependant, le même ensemble admet un autre découpage, en deux volets cette fois, avec une frontière qui passe au beau milieu du discours direct attribué au héros. En effet, un basculement s'opère lorsque Narcisse découvre que la situation est sans espoir, avec l'exclamation iste ego sum («mais tu n'es autre que moi-même !», v. 463).

Or, je l'ai remarqué plus haut ${ }^{59}$, le centon obéit de même à un clivage qui advient en son milieu: (v. 1-8) un personnage se laisse gagner par les délices du site et de la figure qu'il y découvre; (v. 9-16) le récit évoque l'impasse où le héros se trouve, subjugué par sa propre image ${ }^{60}$. Ainsi, le centon se conforme à une double structuration analogue à celle de l'œuvre-cadre: un cheminement narratif en trois étapes se combine avec un contraste sémantique en deux parties.

Certes, l'abrègement drastique et les contraintes de la composition amènent à supprimer les paroles de Narcisse, à travers lesquelles on le voyait prendre conscience d'un dédoublement qui l'accable. Dans le centon, l'élucidation reste l'apanage du narrateur, qui souligne le désastre d'une passion illusoire. Dès lors, il serait vain de chercher un parallélisme strict entre les divers segments de l'histoire dans l'œuvrecadre et le centon. Le décalage marqué de ce dernier répond à une restriction calculée du scénario, permettant un raccourci narratif, apte à produire ce que l'on pourrait 
nommer une diégèse synecdochique: il s'agit de reprendre une partie des événements avec des similitudes capables d'évoquer toute l'extension de l'œuvre-cadre.

Aussi le centon demeure-t-il centré sur les circonstances liées à la découverte de la source et de l'image dans l'eau ${ }^{61}$ : même ses derniers vers comportent certains détails qui rappellent la portion initiale du développement ovidien. En particulier, la mention du reflet, que Narcisse contemple sans en saisir la nature, n'intervient que tardivement (v. 12): l'ablatif imagine rappelle, dans les Métamorphoses, le v. 416, où une forme semblable se retrouve à la même place; plus largement, la situation répond aux apostrophes fictives du narrateur à l'adresse du personnage, comme pour l'avertir de son illusion (v. 432-434) (2. $^{2}$

Cependant, le récit aboutit à un enlisement final qui comporte une difficulté: il manque la circonstance décisive sur laquelle se termine l'histoire ovidienne, la métamorphose. On va s'apercevoir qu'il ne s'agit pas véritablement d'une lacune.

\section{La conclusion de l'aventure}

Les deux derniers vers dans le centon procurent l'impression déceptive d'un inachèvement, même si, d'après l'œuvre-cadre, l'issue fatale ne fait guère de doute. D'ailleurs, le v. 13, quand il mentionne la passion qui dévore Narcisse, rappelle la dernière phase du récit ovidien, où le héros dépérit peu à peu, consumé par ses désirs inaccessibles (M. III, 490). Mais la fin du poème, loin d'aborder l'étape ultérieure de la métamorphose, s'interrompt sur l'inanité d'une action répétitive, le triple effort du héros pour étreindre son reflet (v. 16).

La composition même de cet hexamètre semble hésitante, sinon défaillante, renforçant l'effet d'incomplétude. Pour former le premier hémistiche, deux extraits fusionnent: l'un offre le motif d'une triple tentative, mais il lui manque un verbe conjugué pour s'articuler avec la fin du vers (ter conatus ibi, «à trois reprises, alors, ayant essayé ») ${ }^{63}$; l'autre se rattache sans difficulté au fragment suivant, mais il mentionne un double échec (bis conatus erat, "à deux reprises, il avait essayé ") ${ }^{64}$. Le centoniste, tout en retenant la forme syntaxique adéquate, a préféré, au prix d'une hybridation entre les extraits, maintenir l'adverbe ter, issu du même hexamètre virgilien que le fragment précédent.

C'est que le geste triplé de Narcisse, mis en valeur grâce à l'enjambement entre les v. 15 et 16, se calque sur les deux passages de Virgile, où Enée s'efforce en vain d'embrasser l'ombre fuyante d'un être cher (imago de Créüse durant la chute de Troie, puis d'Anchise aux Enfers) ${ }^{65}$. Or, la réduplication virgilienne trouve un corrélat dans l'épisode ovidien, avec la tentative inutile de la nymphe Écho pour enlacer Narcisse ( $M$. III, 388), puis celle du héros afin de saisir sa propre image au sein des ondes (M. III, 428-429).

La similitude qu'offre le premier de ces passages avec l'hexamètre réitéré dans l'Enéide, en reproduisant l'alliance des noms collo et bracchia, montre qu'Ovide avait ménagé là une réminiscence qui, associant Écho avec un spectre, préfigurait allusivement son évanescence ultime en un pur effet sonore (M. III, 401) ${ }^{66}$. Cette ressemblance n'a pas échappé au centoniste: en mobilisant le vers de Virgile, il a prolongé la similitude grâce à l'extrait suivant qui, bien qu'issu d'un tout autre contexte, présente, de même que chez Ovide, une occurrence du verbe sperare. 
Dans le second passage, le texte des Métamorphoses procède à une variation: il modifie la syntaxe et introduit un enjambement ${ }^{67}$. À son tour, le centon répercute cet agencement spécifique comme permet de le vérifier la comparaison ci-dessous ${ }^{68}$.

centon v. 15-16

et, praeceps animi, collo dare bracchia circum,

ter conatus erat, nec quid speraret habebat.

et dans l'irrépressible élan de son cœur, par trois fois il avait essayé

d'entourer ce cou de ses bras, mais n'avait rien à espérer.

Ovide M. III, 388Ibat ut iniceret sperato bracchia collo.

Elle s'avançait, pleine d'espoir, pour lui jeter les bras autour du cou.

Ovide M. III, 428-429

In mediis quotiens uisum captantia collum

bracchia mersit aquis nec se deprendit in illis!

Que de fois, cherchant à saisir ce cou offert à sa vue,

au milieu des eaux, il a plongé les bras et n'a pu y atteindre!

Ainsi, l'assemblage du v. 16, loin d'être une solution approximative, manifeste une démarche complexe, où la récriture réussirait même à se mettre en scène, au moment de conclure. L'éviction du bis, qu'offrait $E n$. VI, 32, pour adopter le triplement, suggère une lecture métapoétique du résultat. En effet, l'adverbe ter, qui répond au quotiens exclamatif ovidien, serait susceptible d'illustrer discrètement l'ajout par le centon d'une troisième occurrence au dédoublement des Métamorphoses ${ }^{69}$. Mais si le caractère insolite du passage ne relève pas d'une maladresse, l'absence de la métamorphose pourrait ne pas être fortuite. L'insistance sur le manque dans le dernier hémistiche se concevrait comme une incitation ironique à mieux chercher: si elle annonce l'échec, voire la perte de Narcisse, elle ne suffit pas à clore l'aventure en cohérence avec l'œuvre-cadre.

Dès lors, c'est à l'autre extrémité du poème que se reporte l'enquête: on y relève une métaphore d'apparence banale, précisant que le jeune homme introduit par le récit est dans la «fleur » de l'adolescence (v. 1). Or, prise au pied de la lettre, l'image comporte une dimension proleptique latente. Elle préfigure d'autant mieux le devenir floral de Narcisse que le personnage est alors associé à la blancheur, avec l'adjectif initial candida. Or les Métamorphoses insistent sur la teinte immaculée de la fleur née avec la disparition du héros, soulignant l'échange accompli entre son corps et la corolle. On découvre ainsi, comme le montre la comparaison suivante, une remarquable coïncidence entre la phrase initiale du centon et la dernière du récit ovidien: le mot ultime de celui-ci répond même au premier de celui-là ${ }^{70}$.

centon v. 1-2

Candida per siluam primaeuo flore iuuentus

adsidue neniebat.

Au cœur de la forêt le charme d'un jeune homme au teint clair, dans la fleur de son

adolescence

venait se promener bien souvent.

Ovide M. III, 509-510

Nusquam corpus erat; croceum pro corpore florem

inueniunt foliis medium cingentibus albis.

Le corps avait disparu; à sa place, on trouve une fleur couleur de safran,

dont le cœur est entouré de blancs pétales.

Dès lors, le poème, par un effet de circularité, se referme pour ainsi dire grâce à son incipit, apte à préfigurer le destin du héros et le dénouement de l'aventure ovidienne. Mais cette anticipation n'appartient pas en propre au centon: elle préside non moins à 
l'agencement du récit dans les Métamorphoses. En effet, l'épisode s'ouvre sur l'annonce encore énigmatique par Tirésias d'un danger lié à la réflexivité (v. 346-350), débouchant sur le rappel de cette prophétie, avec la notoriété qu'elle confère au devin (v. 511-512). Aussi peut-on envisager la solution, intuitive ou concertée, mise en œuvre par le centoniste pour évoquer la métamorphose, comme un équivalent efficace du prologue et de la structure symétrique ménagés par Ovide. Avec une singulière économie de moyens, le surgissement, dès le premier vers, de flore lié à la blancheur dispense le poème d'une conclusion par avance indiquée $e^{71}$. Il justifie d'ailleurs la bizarrerie de l'abstrait iuuentus, accompagnement obligé dans l'hémistiche prélevé ${ }^{72}$.

Ainsi la structure de l'épisode ovidien fait l'objet d'une transposition qui prouve une connaissance familière des Métamorphoses en même temps qu'elle débouche sur une notable inventivité. Car la présence initiale de la fleur, dans le décor que le centon esquisse, légitime le rapprochement que le début du poème accomplit entre le paysage et le jeune homme, l'un comme l'autre inspirant la passion.

La finesse de certains dispositifs ainsi repérés dans le centon, montrant la prise en compte scrupuleuse du texte ovidien, manifeste un brio qui tranche avec la réputation de médiocrité longtemps attachée au centon de Narcisse ${ }^{73}$. L'apparence d'une facture schématique et d'une extrême réduction par rapport au récit des Métamorphoses lui a sans doute valu des jugements sévères, dispensant d'effectuer un examen plus approfondi ${ }^{74}$. Son intérêt, malgré les indéniables faiblesses qu'il comporte, a été récemment mis en valeur par Scott McGill qui a souligné la sophistication des liaisons que le centoniste établit, dépassant un simple montage d'extraits virgiliens: il indique notamment les interactions sémantiques sous-jacentes entre le poème et les passages mobilisés dans l'œuvre-source, ainsi que certains rappels spécifiques d'Ovide, obtenus avec les énoncés empruntés à Virgile ${ }^{75}$. On va voir qu'il est possible de prolonger un tel repérage et de faire apparaître pour l'ensemble du centon une nébuleuse d'échos.

\section{L'accord d'une double floraison: déclinaison d'échos réfléchis}

52 Juste avant que s'accomplisse, à la charnière du poème, la transition entre l'attirance que le personnage éprouve pour le site et l'amour qui s'empare de lui pour son reflet, le poème rend particulièrement sensible l'impact de l'œuvre-cadre, en accueillant une série d'échos. Ainsi le rapprochement diégétique introduisant dans le décor le double de Narcisse, présenté, à la suite d'Ovide, comme le fils d'une nymphe (v. 8), est-il préparé grâce à plusieurs coïncidences verbales. On voit alors la communauté de formulation se cristalliser sur un passage des Métamorphoses, celui qui, justement, décrit chez Ovide le coup de foudre du héros.

53 J'ai montré la manière dont le centoniste était parvenu à capter l'évocation d'un breuvage métaphorique pour marquer la disposition amoureuse qui d'avance gagne Narcisse (v. 4) ${ }^{76}$. Mais c'est surtout au début du v. 6, qu'est sollicitée la mémoire des lecteurs familiers avec le récit ovidien: l'effet sidérant que la contemplation des lieux procure au personnage se traduit par un verbe, stupet, « il demeure en arrêt », dont on rencontre un composé, adstupet, dans les Métamorphoses, pour marquer l'instant même où le héros découvre sa figure dans l'eau (v. 418) ${ }^{77}$. De plus, le dum qui amorce le vers du 
centon se retrouve juste au-dessus dans le récit d'Ovide: au début des v. 415 et 416 , un dumque récurrent sert à introduire le motif commun de la soif ${ }^{78}$.

En somme, un rapprochement spécifique avec l'œuvre-cadre s'établit, grâce à une stratégie d'écriture fondée sur la sélection dans l'œuvre-source d'un passage auquel renvoyait le récit ovidien. Le phénomène est ici d'autant plus notable que le recours à stupet intervient à deux reprises dans les Métamorphoses, pour indiquer les réactions de Narcisse. En effet, le verbe simple, au même endroit dans l'hexamètre, marque l'étonnement du héros quand il entend Écho répéter ses propres paroles et ne comprend pas d'où provient cette voix (M. III, 381) ${ }^{79}$.

Dans un cas comme dans l'autre, le héros chez Ovide est confronté à un phénomène de redoublement où lui-même est impliqué, tout comme Enée, dans l'extrait de Virgile, découvrait avec saisissement, sur le temple de Junon à Carthage, la représentation de scènes qu'il avait vécues, et jusqu'à sa propre image $(E n \text {. I, } 488)^{80}$. La réduplication de la forme verbale en écho se conjugue avec la correspondance thématique pour affermir dans le récit ovidien la réminiscence virgilienne.

Le centoniste a su discerner l'impact de ce dispositif. La preuve en est qu'il outrepasse un rappel isolé, en construisant le v. 6 grâce à deux extraits issus de la même scène dans l'Enéide (I, $495+464)$ : de la sorte, il reproduit la démarche d'Ovide qui, dans les v. 418-419, a conjugué le souvenir de deux hexamètres virgiliens appartenant à ce passage et mettant chacun l'accent sur la surprise d'Enée, d'abord devant les peintures, puis quand il voit arriver ses compagnons qu'il croyait morts dans la tempête (En. I, 495 et $513)^{81}$. On peut, en observant les extraits suivants, mieux saisir les données de cette récriture, dont le centon relance le processus ${ }^{82}$.

En. I, 495

dum stupet obtutuque haeret defixus in uno

tandis qu'il demeure en arrêt, figé dans cette seule contemplation

En. I, 513

obstipuit simul ipse, simul percussus Achates

Aussitôt Enée lui-même resta figé, ainsi qu'Achate, bouleversé

M. III, 381

Hic stupet utque aciem partes dimittit in omnes

Lui demeure en arrêt et, après avoir promené de tous côtés ses regards

M. III, 416, 418-419

Dumque bibit, uisae correptus imagine formae

Adstupet ipse sibi uultuque immotus eodem

Haeret ut e Pario formatum marmore signum

Tandis qu'il boit, saisi par la vue de sa beauté qui se reflète...

Il s'extasie face à lui-même, demeure immobile, le visage figé,

comme une statue taillée dans le marbre de Paros

Centon 6 (En. I, $495+\mathrm{I}, 464$ )

Dum stupet atque animum pictura pascit inani

Mais tandis qu'il demeure en arrêt, gavant son cœur d'un spectacle illusoire

Avec cet ensemble complexe de corrélations se dégage, en quelque sorte, une circularité de l'imitatio, puisque les fragments de Virgile réunis dans le v. 6, interviennent comme en rappel de passages repérables chez l'un de ses successeurs. En l'occurrence, un extrait de l'Enéide sert, paradoxalement, à récrire les Métamorphoses, comme si l'épopée virgilienne était tributaire d'une œuvre pourtant composée près de trois décennies après elle. Le centoniste, montrant une intuition aigüe des mécanismes 
qui opèrent dans l'œuvre-cadre, en reproduit autant que possible les particularités grâce aux matériaux qu'il parvient à mobiliser dans l'œuvre-source.

58 À cet égard, le début du v. 6 apparait comme un point de repère décisif: il implique une scène marquante de l'Enéide, elle-même évoquée dans le récit ovidien par un double écho. De la sorte, il offre un signal de la récriture qui se trame dans le centon: elle se fonde sur le récit des Métamorphoses non seulement comme une base diégétique mais aussi comme une référence textuelle. Un phénomène de coalescence en découle, où le choix des extraits dans l'œuvre-source est en partie déterminé par le retentissement qu'ils ont pu avoir dans l'œuvre-cadre.

Cette orientation se confirme avec le v. 7, qui décrit les regards lancés par Narcisse à son image: la formule expleri mentem nequit, « son esprit ne peut se rassasier ", provient de En. I, 713, où Didon contemple l'Amour qui a pris les traits de Iule. On rencontre chez Ovide un énoncé approchant, avec l'adjectif inexpleto qui sert à qualifier l'œil insatiable du personnage, rivé sur son reflet (M. III, 439) ${ }^{83}$. Le lien, quoique ténu en apparence, est rendu plus aisément perceptible, grâce à la commune indication du regard, caractérisée par un ablatif instrumental ${ }^{84}$.

60 Mais c'est plus loin, dans le v. 14, que se manifeste avec une netteté particulière le retour sous-jacent de tournures ovidiennes parmi l'assemblage des extraits virgiliens. Pour accentuer l'effet, le même passage des Métamorphoses est encore mobilisé: "l'espoir chimérique », uana spe, rappelle le début du v. 417; la formule indiquant la fixation de Narcisse « sur son propre visage », ipsius in uultu, recoupe celle du v. 418 qui, évoquant la réflexivité, associe le pronom ipse avec l'ablatif uultu ${ }^{85}$. Un jalon supplémentaire advient grâce au participe amantem qui clôt le vers: répondant à la forme amat du v. 417, il coïncide surtout avec la fin du v. 447, dans le discours que Narcisse tient peu après ${ }^{86}$.

61 Ce vers 14 est donc propre à susciter une impression diffuse de retrouvaille, même si l'on n'appréhende pas aussitôt le détail de ses ressemblances avec le texte ovidien. D'ailleurs, dans le v. 12, un plus discret accord prédispose un tel effet: miratur qui désigne chez Virgile l'ébahissement d'Enée en contemplant sur son bouclier les scènes qu'a représentées Vulcain, reproduit une occurrence qui, chez Ovide, marque l'admiration de Narcisse pour sa propre beauté (v. 424) ${ }^{87}$. De nouveau, comme avec stupet au v. 6, une forme verbale virgilienne réemployée par Ovide ponctue le centon d'une réminiscence: la progression dans le poème fait passer de la sidération à une posture contemplative ${ }^{88}$.

62 En confrontant les multiples formules qui recoupent celles des Métamorphoses, on s'aperçoit qu'elles obéissent à une double cohérence: elles se rattachent au thème de la subite passion pour une image, et corrélativement se relient à une portion du récit ovidien, dont l'ampleur est du même ordre que celle du centon, la scène où le héros tombe amoureux de son reflet (M. III, 413-440).

Un tel constat n'a rien d'étonnant, puisque l'on a vu que, dans le centon, le déroulement $\mathrm{du}$ récit se figeait pour ainsi dire, limitant la diégèse à cette étape, où émergent il est vrai les principaux enjeux de la fiction: c'est au cours de cette phase que s'ouvre une faille dans l'assurance du héros, que son identité vacille, quand il se laisse piéger par un désir impossible à satisfaire. En accumulant les ressemblances avec cette partie de l'épisode, le centon rend d'autant plus sensible la récriture dont il procède ${ }^{89}$. Du coup, les similitudes verbales qui s'établissent avec l'œuvre-cadre, sans suivre un 
ordre absolument identique, coïncident avec le retour des aspects qui caractérisent la situation ${ }^{90}:$ l'acte de boire comme déclencheur d'un amour qui produit une sidération (v. 3-6); l'espoir trompeur d'un amant captif de sa propre image (v. 12-14); l'admiration pour une beauté qui n'est celle que d'un reflet (v. 9-12); la vaine tentative pour happer l'être aimé (v. 15-16); liée à la métaphore du feu, la relance du désir par les regards (v. 7-8).

Seuls, les deux premiers vers ne s'inscrivent pas dans l'ensemble ainsi dégagé: c'est qu'ils jouent un rôle à part dans la structure du centon, avec l'annonce latente de la métamorphose, ce qui, chez Ovide, les rattache aux deux derniers vers du récit ${ }^{91}$. À l'opposite, ce sont les v. 15-16 qui offrent, dans le domaine du phrasé, la ressemblance la plus nette avec le texte des Métamorphoses, comme pour marquer un point d'orgue, à partir duquel la lecture, s'interrogeant sur le coup d'arrêt qu'elle subit, va se trouver reconduite vers l'amorce du poème. Sur ce plan, au v. 15, la périphrase virgilienne pour l'acte d'embrasser, collo dare bracchia circum, semble particulièrement savoureuse, avec l'insistance qu'elle fait porter sur la circularité.

En outre, le centoniste, sensible à l'affinité qu'établit le récit d'Ovide entre les deux personnages d'Écho et de Narcisse, rémunère, pour ainsi dire, le défaut de parallélisme diégétique: s'il omet toute référence à la nymphe, l'aventure de celle-ci n'est pas pour autant absente. En effet, nombre de réminiscences ovidiennes se soldent par une double référence à des morceaux concomitants, l'un dédié à la passion d'Écho, l'autre à celle de l'adolescent. C'est le cas pour la périphrase virgilienne du v. 15 dont Ovide s'est inspiré à deux reprises (v. 388 et $428-429)^{92}$.

Le phénomène se renouvelle avec la mention du reflet, imagine (v. 12), qui reprend, à la même forme et la même place, un terme dédoublé chez Ovide, d'abord pour indiquer un écho (v. 385), puis une image répercutée dans l'eau (v. 416) ${ }^{93}$. En l'occurrence, comme ce nom synthétise chez Ovide la réduplication et se trouve lui-même redoublé, il acquiert dans le centon un relief particulier. Chez Virgile (En. VIII, 730), il désignait une simple image, mais avec l'intégration du fragment dans un contexte distinct, il s'entend désormais comme un miroitement. Son articulation avec le verbe gaudet montre un personnage qui prend plaisir à contempler un reflet dont il méconnaît la nature. Cet attrait, que l'écho dédoublé avec les Métamorphoses associe au rappel des pièges auditif et visuel dramatisés par Ovide, ne concerne pas forcément que les circonstances immédiates de l'intrigue: il s'applique potentiellement à la nature réflexive du centon, elle qui, par définition, échappe au personnage.

En somme, la fascination pour le rapport paradoxal du reflet à l'unicité de l'être qu'il réplique est apte à désigner de façon implicite l'agrément d'un exercice poétique ajustant des morceaux pour produire le double d'une fiction antérieure. Ce faisant, le centoniste, même s'il n'évoque pas directement Écho dans son récit, a tenu compte indéniablement de la dualité qui gouverne l'épisode ovidien, où les aventures solidaires de deux êtres concernant chacune le motif de la réduplication se trouvent prises dans un rapport spéculaire ${ }^{94}$.

Sa fidélité à la démarche d'Ovide se manifeste aussi dans sa façon d'employer à deux reprises l'image de la flamme, pour évoquer la passion (ardescit, v. 7 et ignis edax, v. 13). Cette métaphore est empruntée par Ovide à l'Enéide où elle s'applique à Didon ${ }^{95}$. Elle souligne d'abord le désir de la nymphe à la vue de Narcisse (M. III, 372-374), puis se rapporte à l'adolescent amoureux de lui-même, selon deux étapes: l'ardeur que suscitent au début ses regards (v. 426 et 430$)^{96}$, et plus tard la manière dont cet amour le 
dévore (v. 490) ${ }^{97}$. Le centon adapte donc ce dispositif: la figure, au v. 7, rappelle chacun des passages ovidiens couplés, illustrant pour Écho et Narcisse les atteintes d'un feu nouveau; au v. 13, elle est exclusivement liée à Narcisse, à la phase où le héros dépérit ${ }^{98}$.

Par la même occasion, le redoublement de la métaphore ignée joue un rôle décisif pour la cohésion du poème, en reliant l'un à l'autre les passages concernés. Mais le début du v. 13 ajoute un détail notable, avec l'adjectif edax («dévorant»), qui introduit une image supplémentaire, renforçant l'association avec le développement précédent. En effet, celui-ci file une métaphore qui rattache passion et appétit, avec les formes verbales pascit ("gaver », v. 6), puis expleri («rassasier», v. 7) ${ }^{99}$. L'ensemble prolonge par analogie la métaphore du v. 4 , où déjà selon une adaptation du modèle ovidien, le spectacle du locus amoenus est appréhendé comme un breuvage (bibebat). C'est donc un régime figural qui se dégage dans le centon. Simplement la juxtaposition des métaphores aux v. 6-7 fait place à leur articulation au début du v. 13: l'insatiable appétence agissait comme un feu intérieur; elle est désormais une flamme qui absorbe l'être. De la sorte est transposé le dépérissement du Narcisse ovidien ${ }^{100}$.

70 Mais l'agencement propre au centon coïncide avec la mise en place d'une tonalité sémantique analogue à celle des Métamorphoses, grâce à un jeu sur les contrastes aboutissant à un oxymore où l'on reconnaît une approche ovidienne ${ }^{101}$. Cela se traduit d'abord, à la charnière des v. 6 et 7, par la juxtaposition de l'adjectif inani avec le verbe expleri, termes qui, pris au pied de la lettre, dénotent successivement le vide et le plein $^{102}$. Le phénomène se répète ensuite dans le v. 13, réunissant les termes antithétiques $\mathrm{du}$ feu et des eaux ${ }^{103}$. De la sorte, en inversant l'ordre des termes, cet hexamètre accueille un agencement analogue à celui d'Ovide, au v. 490, où le verbe initial, liquitur, « il se liquéfie », s'oppose au nom final, igni ${ }^{104}$.

71 On découvre ainsi que le texte fonctionne selon un double régime: d'une part, il promeut une adaptation fidèle des données qui caractérisent le récit ovidien; d'autre part, il acquiert une autonomie relative en s'appuyant sur un choix de fragments qui permet d'établir des connexions supplémentaires entre ses composantes ${ }^{105}$. Une telle stratégie d'écriture intrinsèque n'est nullement antinomique à la mise en place de concordances avec l'œuvre-cadre. Elle procède d'un même jeu pour dépasser la simple application de la contrainte centonale, réduite à l'assemblage de segments débouchant sur une signification cohérente.

72 Bref, le florilège, issu d'une cueillette parmi les hexamètres virgiliens, manifeste une expérimentation poussée de l'écriture poétique, soutenue par une connaissance fine des Métamorphoses qui autorise, avec l'appoint varié de l'analogie, maints rappels des formes et des coloris propres au bouquet ovidien.

73 En fin de compte, la lecture des Métamorphoses pratiquée par le centoniste, telle que mon analyse a tenté de la reconstituer, impressionne par son alliance de rigueur et de souplesse. Elle semble se fonder sur le présupposé chez ses destinataires non seulement d'une connaissance détaillée de l'anecdote mais d'une fréquentation familière de l'œuvre. C'est pourquoi l'écriture admet l'emploi de périphrases, le recours à une diégèse elliptique, se suffit d'un mot liminaire pour signifier la métamorphose. En même temps, le centoniste multiplie les signaux d'une connivence avec les détails d'une œuvre-cadre, dont il est parfois obligé néanmoins de s'écarter amplement.

De fait, pas question de récrire dans le détail l'épisode ovidien sur la base des exigences propres au genre pratiqué: le centon de Narcisse permet de goûter la reprise très 
distanciée d'un texte, de sentir au moins diffusément les similitudes ponctuelles dont elle est constellée, de savourer le jeu stratifié d'un palimpseste qui fait écrire Virgile à la manière d'Ovide ${ }^{106}$.

Certes, le résultat n'offre pas toujours une limpidité ni un lissage parfaits. Il arrive que le grain de l'écrit se donne à percevoir avec une certaine rudesse: les aspérités dans le phrasé, les énoncés au laconisme excessif, qui jalonnent le centon, peuvent être conçus comme le tribut imposé pour que l'enchaînement représentatif se poursuive en dépit des difficultés. D'ailleurs, dans ses réussites mêmes, le genre atteste la minutie des opérations qui l'échafaudent en se manifestant comme un exercice virtuose de montage. Antonio Cataldo affirme à juste titre la visibilité de l'effort consenti par le centoniste pour adapter les morceaux qu'il mobilise: "par elle-même la structure extérieure des centons amène à éprouver le déroulement de l'action dans le poème comme étroitement dépendant de tous ses éléments constitutifs qui, d'une manière ou d'une autre, impliquent un recours à la réflexion ${ }^{107} »$.

Bref, ce type de poème affiche en sa facture l'artificialité qui le fonde. Le travail de composition s'en trouve démystifié, en même temps que les ressources disponibles et les trouvailles effectuées deviennent appréhendables. Comme le remarque Françoise Desbordes, «le centon, cousu de fil blanc, expose les secrets de fabrication »; par suite, donnant accès aux manipulations qu'a dû accomplir l'écrivain, il court le risque de scandaliser, étant donné que, d'habitude, «le principal effort des littérateurs est de cacher le jeu, leur jeu: dissimuler les rouages ${ }^{108} »$.

En somme, on est confronté à une œuvre qui vaut d'abord pour elle-même, non pour le message qu'elle délivre, ni la sensibilité qu'elle exprime: elle n'a d'autre vocation que de bâtir au mieux l'édifice qu'elle institue, de réaliser ainsi le double programme qu'elle s'assigne envers l'œuvre-source et l'œuvre-cadre.

On comprend dès lors quelles réticences cette sorte de poème peut provoquer chez ceux qui, dans la littérature, recherchent de manière exclusive la formulation d'une pensée ou l'évocation mimétique d'événements ${ }^{109}$. Selon cette approche, l'écrit se doit de faire oublier les éléments qui le constituent, de manière à ne pas relativiser la prégnance des effets de sens. Au contraire, le centon livre d'emblée les ressorts de sa structure fragmentaire et dérivée, en se posant comme un conglomérat d'emprunts: il met ainsi l'accent sur un travail d'ajustement qui produit à mesure la signification, excluant l'originalité pure, puisqu'il réutilise des énoncés préalablement formés. Ce processus, quand on accepte d'en tenir compte, suppose que l'on envisage la représentation comme le résultat d'un ordre qui l'excède, puisqu'elle doit se plier à des règles préalables ${ }^{110}$.

79 À cet égard, la pratique du centon se place à sa manière dans la lignée de la démarche ovidienne. En effet, l'édifice que constituent les Métamorphoses s'est élaboré comme une sorte de patchwork mythologique, grâce à des prélèvements dans la légende, souvent limités à un épisode qui débouche sur une métamorphose, puis articulés en un récit global dont l'agencement répond à une contrainte diégétique unitaire. D'une certaine façon, les extraits seraient à l'anecdote du centon ce que les aventures mythiques sont à cette diégèse unifiée $e^{111}$.

80 Reprendre la fable des Métamorphoses consacrée au reflet était pour le centoniste un choix opportun. Le mérite de cet inconnu est d'avoir traité son modèle avec une approche lucide, en s'attachant non seulement à transposer de façon suggestive la diégèse, mais à démêler certains ressorts plus fins de l'œuvre-cadre. Cette démarche 
ingénieuse, témoignage d'une culture raffinée au cœur de l'Antiquité tardive, offre aux lecteurs modernes un regard indirect sur la poétique d'Ovide. Elle est aussi l'occasion d'appréhender les enjeux de la récriture, telle que la pratiquaient les poètes latins, en considérant le centon comme le passage à la limite d'une démarche qu'annonçait, dès l'époque augustéenne, la physionomie composite de certains poèmes où foisonnent les réminiscences, comme si bien des vers reposaient sur les formules redistribuées d'œuvres poétiques antérieures.

\section{Annexe 1}

\section{Le centon anonyme de Narcisse}

\begin{tabular}{|c|c|c|}
\hline Candida per siluam / primaeuo flore iuuentus & & [En VIII, $82+E n$ VII, 162] \\
\hline adsidue ueniebat. Ibi haec / caelestia dona & & {$[B \mathrm{II}, 4+G \mathrm{IV}, 1]$} \\
\hline et fontes sacros / insigni laude ferebat, & & {$[B \mathrm{I}, 52+E n \mathrm{I}, 625]$} \\
\hline insignis facie, / longumque bibebat amorem, & & {$[E n$ IX, $583+E n \mathrm{I}, 749]$} \\
\hline intentos uoluens oculos, / securus amorum. & 5 & [En VII, $251+E n \mathrm{I}, 350]$ \\
\hline Dum stupet / atque animum pictura pascit inani, & & {$[E n \mathrm{I}, 495+E n \mathrm{I}, 464]$} \\
\hline expleri mentem nequit ardescitque tuendo & & {$[E n \mathrm{I}, 713]$} \\
\hline egregium forma iuuenem, / quem nympha crearat: & & [En VI, 861=XII, $275+E n \mathrm{X}, 551]$ \\
\hline sic oculos, sic ille manus, sic ora ferebat. & & [En III, 490] \\
\hline His amor unus erat, / dorso dum pendet iniquo, & 10 & {$[E n \mathrm{IX}, 182+E n \mathrm{X}, 303]$} \\
\hline oblitusue sui est / et membra decora iuuentae & & {$[E n$ III, $629+E n$ IV, 559$]$} \\
\hline miratur rerumque ignarus imagine gaudet. & & [En VIII, 730] \\
\hline Ilicet ignis edax / secreti ad fluminis undas & & [En II, $758+E n$ III, 389] \\
\hline ipsius in uultu / uana spe lusit amantem & & {$[G \mathrm{I}, 452+E n \mathrm{I}, 352]$} \\
\hline et, praeceps animi, / collo dare bracchia circum & 15 & [En IX, $685+E n$ II, 792=VI, 700] \\
\hline ter conatus erat, / nec quid speraret habebat. & & [En VI, 32/II, 792=VI, $700+$ B II, 2] \\
\hline
\end{tabular}




\section{BIBLIOGRAPHIE}

Ausone, CEuvres en vers et en prose, traduction nouvelle de Max Jasinsky, tome 1, Paris, 1934.

Bright, David F., « Theory and practice in the Vergilian Cento », Illinois Classical Studies, 9, 1984, pp. 79-90.

Cataldo, Antonio, « Maro mutatus in melius (Espedienti compositivi nel centone virgiliano di Proba)», Quaderno - Istituto di lingue e letterature classiche, Lecce, 1, 1980, pp. 17-60.

Desbordes, Françoise, « Le corps étranger - Notes sur le centon en général et la Médée d'Hosidius Géta en particulier ", Argonautica - Trois études sur l'imitation dans la littérature antique, Bruxelles, 1979, pp. 83-108.

Dumarsais, Des tropes ou des différents sens, présentation, notes et traduction Françoise DrouaySoublin, Paris, 1988.

Ellsworth, James D., « The episod of the Sibyl in Ovid's Metamorphoses (14.103-56) », East meets West: homage to Edgar C. Knowlton, edited by Roger L. Hadlich, J. D. Ellsworth, Honolulu, 1988, pp. 47-55.

Galand-Hallyn, Perrine, Le Reflet des fleurs - Description et métalangage poétique d'Homère à la Renaissance, Genève, 1994.

Genette, Gérard, Figures III, éditions du Seuil, Paris, 1972.

Genette, Gérard, Métalepse, éditions du Seuil, Paris, 2004.

Grangaud, Michelle, « Sartre poète », Conséquences, 5, 2001, pp. 12-17.

Green, R. P. H., The works of Ausonius, Oxford, 1991.

Hilgard, Alfred, Grammatici Graeci, Part I, Vol. II, Hildesheim, (1901), 1979.

Lamacchia, Rosa, « Problemi di interpretazione semantica in un centone virgiliano », Maia, 10, 1958, pp. 161-188.

McGill, Scott, « Tragic Vergil: rewriting Vergil as a tragedy in the cento Medea », Classical World, 95, 2002, pp. 143-161.

McGill, Scott, Virgil recomposed - The mythological and secular centos in Antiquity, Oxford, 2005.

Palla, Roberto, « Risvolti di tecnica centonaria », Civiltà classica e cristiana, 4, 1983, pp. 279-297.

Pavlovskis, Zoja, « Statius and the late epithalamia », Classical Philology, 60, 1965, pp. 164-177.

Petrone, Gianna, « Locus amoenus/locus horridus: due modi di pensare la natura ", L'Uomo antico e la natura, Atti del convegno nazionale di studi, a cura di Renati Uglione, Torino, 1998, pp. 177-195.

Petrucci, Armando, « Virgilio nella cultura scritta romana », Virgilio e noi, Genova, 1981, pp. 51-72.

Polara, Giovanni, « I Centoni », Lo Spazio letterario di Roma antica, vol. III, La Ricezione del testo, Roma, 1990, pp. 245-275.

Riese, Alexander, Anthologia Latina I.1, Leipzig, 1894.

Rosati, Gianpiero, Narciso e Pigmalione - Illusione e spettacolo nelle Metamorfosi di Ovidio, Firenze, 1983. 
Salanitro, Giovanni, Osidio Geta: Medea, introduzione, testo critico, traduzione e indici. Con un profilo della poesia centonaria greco-latina, Roma, 1981.

Salanitro, Giovanni, « Osidio Geta e la poesia centonaria », Aufstieg und Niedergang der römischen Welt, Part II, 34.3, Berlin - New York, 1997, pp. 2314-2360.

Salanitro, Giovanni, Silloge dei Vergiliocentones minori, Roma, 2009.

Schiavetta, Bernardo, «Un éclat de ta voix », Conséquences 5, 2001, pp. 36-38.

Tertullien, Traité de la prescription contre les hérétiques, texte et traduction de R. F. Refoulé, P. De Labriolle, Paris, 1957.

Tronchet, Gilles, La Métamorphose à l'œuvre, Louvain - Paris, 1998.

Tronchet, Gilles, « À la lumière d'un rêve obscur - Ovide, am. III, 5 et la composition d'un emblème élégiaque », FuturAntico, 6, 2007, pp. 145-195.

Vallozza, Maddalena, « Rilievi di tecnica compositiva nei centoni tramandati con la Medea dal codice Salmasiano », Studi in onore di Adelmo Baragazzi, Roma, 1986, vol. 1, pp. 335-341.

\section{ANNEXES}

\section{Traduction}

$\mathrm{Au}$ cœur de la forêt le charme d'un jeune homme au teint clair, dans la fleur de son adolescence, venait se promener bien souvent. Là, il célébrait par d'insignes louanges les divins agréments de l'endroit, avec sa fontaine sacrée; lui-même d'une insigne beauté, il buvait à longs traits la passion, dardant alentour ses regards, sans craindre de tomber amoureux.

Mais tandis qu'il demeure en arrêt, gavant son cœur d'un spectacle illusoire, son esprit ne peut se rassasier; et voici qu'il s'enflamme en contemplant un jeune homme extraordinairement beau, dont la mère était une nymphe: celui-ci avait les mêmes yeux, les mêmes mains, le même visage que les siens.

Un seul amour les unissait, tandis qu'il restait suspendu sur la berge rude; à moins qu'il ait oublié sa propre identité, quand il s'émerveille d'un corps aux grâces juvéniles, et que, sans comprendre la situation, il se plaise à un reflet.

Les flammes d'une passion dévorante, au bord d'un cours d'eau, en un lieu isolé, eurent tôt fait de leurrer un amant épris de son propre visage, en lui offrant un espoir chimérique, et dans l'irrépressible élan de son cœur, par trois fois il avait essayé d'entourer ce cou de ses bras, mais n'avait rien à espérer. 


\section{Annexe 2}

\section{Principales correspondances thématiques et verbales du centon avec l'épisode d'Écho et Narcisse dans les Métamorphoses (III, 339-510)}

Le graissage fait ressortir les correspondances verbales (termes communs ou semblables).

Le soulignement indique des correspondances thématiques sans retour des mêmes mots.

\begin{tabular}{|c|c|}
\hline - Candida per siluam primaeuo flore iuuentus & $\underline{\text { centon }} \underline{1-2}$ \\
\hline \multicolumn{2}{|l|}{ adsidue ueniebat. Ibi haec caelestia dona } \\
\hline ...poteratque puer iuuenisque uideri. & Ovide M. III, 352-355 \\
\hline \multicolumn{2}{|l|}{ Multi illum iuuenes, multae cupiere puellae; } \\
\hline \multicolumn{2}{|l|}{ sed (fuit in tenera tam dura superbia forma) } \\
\hline ...Narcissum per deuia rura uagantem & Ovide M. III, 370 \\
\hline Siluaque sole locum passura tepescere nullo & Ovide M. III, 412 \\
\hline ...in niueo mixtum candore ruborem & Ovide M. III, 423 \\
\hline Nusquam corpus erat; croceo pro corpore florem & Ovide M. III, 509-510 \\
\hline \multicolumn{2}{|l|}{ inueniunt foliis medium cingentibus albis } \\
\hline - et fontes sacros insigni laude ferebat, & centon $\underline{3-6}$ \\
\hline \multicolumn{2}{|l|}{ insignis facie, longumque bibebat amorem, } \\
\hline \multicolumn{2}{|l|}{ intentos uoluens oculos, securus amorum. } \\
\hline \multicolumn{2}{|l|}{ Dum stupet atque animum pictura pascit inani, } \\
\hline 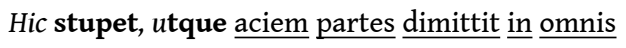 & Ovide M. III, 381 \\
\hline Hic puer et studio uenandi lassus et aestu & Ovide M. III, 413-418 \\
\hline \multicolumn{2}{|l|}{ procubuit faciemque loci fontemque secutus. } \\
\hline \multicolumn{2}{|l|}{ Dumque sitim sedare cupit, sitis $\underline{\text { altera }} \underline{\text { creuit; }}$} \\
\hline \multicolumn{2}{|l|}{ dumque bibit, uisae correptus imagine formae } \\
\hline spem sine corpore amat; corpus putat esse quod & \\
\hline
\end{tabular}




\begin{tabular}{|c|c|}
\hline Adstupet ipse sibi uultuque immotus eodem & \\
\hline - expleri mentem nequit ardescitque tuendo & centon 7-8 \\
\hline \multicolumn{2}{|l|}{ egregium forma iuuenem, quem nympha crearat: } \\
\hline ...Enixa est utero pulcherrima pleno & Ovide M. III, 344-345 \\
\hline \multicolumn{2}{|l|}{ infantem nymphe, iam tunc qui posset amari } \\
\hline ...poteratque puer iuuenisque uideri. & Ovide M. III, 352-355 \\
\hline \multicolumn{2}{|l|}{ Multi illum iuuenes, multae cupiere puellae; } \\
\hline \multicolumn{2}{|l|}{ sed (fuit in tenera tam dura superbia forma) } \\
\hline quoque magis sequitur, flamma propiore calescit & Ovide M. III, 372 \\
\hline Dumque petit petitur pariterque accendit et ardet. & Ovide M. III, 426 \\
\hline Quid uideat nescit; sed quod uidet, uritur illo & Ovide M. III, 430 \\
\hline spectat inexpleto mendacem lumine formam & Ovide M. III, 439 \\
\hline - sic oculos, sic ille manus, sic ora ferebat. & centon $\underline{9-12}$ \\
\hline \multicolumn{2}{|l|}{ His amor unus erat, dorso dum pendet iniquo, } \\
\hline \multicolumn{2}{|l|}{ oblitusue sui est et membra decora iuuentae } \\
\hline \multicolumn{2}{|l|}{ miratur rerumque ignarus imagine gaudet. } \\
\hline Perstat et alternae deceptus imagine uocis & Ovide M. III, 385 \\
\hline dumque bibit, uisae correptus imagine formae & Ovide M. III, 416 \\
\hline Spectat, $\underline{\text { humi }}$ positus, geminum, sua lumina, sidus & Ovide M. III, 420-425 \\
\hline \multicolumn{2}{|l|}{ et dignos Baccho, dignos Apolline crines, } \\
\hline \multicolumn{2}{|l|}{ impubesque genas et eburnea colla decusque } \\
\hline \multicolumn{2}{|l|}{ oris et in niueo mixtum candore ruborem } \\
\hline \multicolumn{2}{|l|}{ cunctaque miratur quibus est mirabilis ipse. } \\
\hline \multicolumn{2}{|l|}{ Se cupit imprudens... } \\
\hline Nunc duo concordes anima moriemur in una & Ovide M. III, 473 \\
\hline
\end{tabular}




\begin{tabular}{|c|c|}
\hline - Ilicet ignis edax $\underline{\text { secreti ad fluminis undas }}$ & centon $\underline{13-14}$ \\
\hline \multicolumn{2}{|l|}{ ipsius in uultu uana spe lusit amantem } \\
\hline Fons erat inlimis, nitidis argenteus undis & Ovide M. III, 407-409 \\
\hline \multicolumn{2}{|l|}{ quem neque pastores, neque pastae monte capellae, } \\
\hline \multicolumn{2}{|l|}{ contigerant... } \\
\hline spem sine corpore amat; corpus putat esse quod unda est. & Ovide M. III, 417-418 \\
\hline \multicolumn{2}{|l|}{ Adstupet ipse sibi uultuque immotus eodem } \\
\hline ...tantus tenet error amantem. & Ovide M. III, 447 \\
\hline Spem mihi nescio quam uultu promittis amico & Ovide M. III, 457 \\
\hline Liquitur et tecto paulatim carpitur igni & Ovide M. III, 490 \\
\hline - et, praeceps animi, collo dare bracchia circum & centon $\underline{15-16}$ \\
\hline \multicolumn{2}{|l|}{ ter conatus erat, nec quid speraret habebat. } \\
\hline Ibat ut iniceret sperato bracchia collo. & Ovide M. III, 388 \\
\hline In mediis quotiens uisum captantia collum & Ovide M. III, 428-429 \\
\hline bracchia mersit aquis nec se deprendit in illis! & \\
\hline
\end{tabular}

\section{Annexe 3}

\section{Corrélations notables du centon avec un passage des \\ Métamorphoses \\ (III, 413-440)}

Le graissage fait ressortir les correspondances verbales (termes communs ou semblables) $)^{112}$.

Le soulignement indique des correspondances thématiques sans retour des mêmes mots.

- centon 3-6

et fontes sacros insigni laude ferebat,

insignis facie, longumque bibebat amorem,

intentos uoluens oculos, securus amorum.

Dum stupet atque animum pictura pascit inani,

Là, il célébrait par d'insignes louanges les divins agréments de l'endroit, avec sa

fontaine sacrée;

lui-même d'une insigne beauté, il buvait à longs traits la passion, dardant alentour 
ses regards, sans craindre de tomber amoureux.

Mais tandis qu'il demeure en arrêt, gavant son cœur d'un spectacle illusoire

- centon 12-14

miratur rerumque ignarus imagine gaudet.

Ilicet ignis edax secreti ad fluminis undas

ipsius in uultu uana spe lusit amantem

il s'émerveille et, sans comprendre la situation, il se plaît à un reflet.

Les flammes d'une passion dévorante, au bord d'un cours d'eau, en un lieu isolé, eurent tôt fait

de leurrer un amant épris de son propre visage, en lui offrant un espoir chimérique

- Ovide M. III, 413-419

Hic puer et studio uenandi lassus et aestu

procubuit faciemque loci fontemque secutus.

Dumque sitim sedare cupit, sitis altera creuit;

dumque bibit, uisae correptus imagine formae

spem sine corpore amat; corpus putat esse quod unda est.

Adstupet ipse sibi uultuque immotus eodem

haeret, ut e Pario formatum marmore signum.

Là s'étendit le jeune homme, fatigué à la fois par la chasse menée avec ardeur et la forte chaleur,

séduit par la figure du site avec sa source.

Tandis qu'il voulait étancher sa soif, une soif nouvelle a surgi en lui;

tandis qu'il boit, fasciné par l'image qu'il voit de sa beauté,

il caresse un espoir sans consistance; il prend pour un corps ce qui n'est que de l'eau.

Il s'extasie face à lui-même, demeure immobile, le visage

figé, comme une statue taillée dans le marbre de Paros.

- centon 7, 9-12

expleri mentem nequit ardescitque tuendo

sic oculos, sic ille manus, sic ora ferebat.

His amor unus erat, dorso dum pendet iniquo,

oblitusue sui est et membra decora iuuentae

miratur rerumque ignarus imagine gaudet.

son esprit ne peut se rassasier; et voici qu'il s'enflamme en contemplant [...]

celui-ci avait les mêmes yeux, les mêmes mains, le même visage que les siens.

Un seul amour les unissait, tandis qu'il restait suspendu sur la berge rude;

à moins qu'il ait oublié sa propre identité, quand il s'émerveille d'un corps aux grâces

juvéniles, et que, sans comprendre la situation, il se plaise à un reflet.

- Ovide M. III, 420-426

Spectat, humi positus, geminum, sua lumina, sidus

et dignos Baccho, dignos Apolline crines,

impubesque genas et eburnea colla decusque

oris et in niueo mixtum candore ruborem

cunctaque miratur quibus est mirabilis ipse.

Se cupit imprudens et qui probat ipse probatur,

dumque petit petitur pariterque accendit et ardet.

Allongé sur le sol, il contemple l'astre double de ses yeux,

ses cheveux dignes de Bacchus et tout aussi bien d'Apollon,

ses joues lisses, son cou d'ivoire, sa bouche charmante

et son teint où le sang tempère une blancheur de neige.

Il admire tout ce qui le rend digne d'être admiré.

Sans prendre garde, il se désire et celui qui est attiré attire lui-même;

il est le sujet et l'objet de la quête, il brûle des feux qu'il suscite en même temps.

centon 15-16

et, praeceps animi, collo dare bracchia circum 
ter conatus erat, nec quid speraret habebat.

et dans l'irrépressible élan de son cœur, par trois fois il avait essayé

d'entourer ce cou de ses bras, mais n'avait rien à espérer.

- Ovide M. III, 428-429

In mediis quotiens uisum captantia collum

bracchia mersit aquis nec se deprendit in illis!

Que de fois, cherchant à saisir ce cou offert à sa vue, au milieu

des eaux, il a plongé les bras et n'a pu y atteindre!

- centon 7-8expleri mentem nequit ardescitque tuendo

egregium forma iuuenem, quem nympha crearat:

son esprit ne peut se rassasier; et voici qu'il s'enflamme en contemplant

un jeune homme extraordinairement beau, dont la mère était une nymphe.- Ovide

M. III, 439

spectat inexpleto mendacem lumine formam

d'un œil que rien ne peut rassasier, il admire son apparence mensongère

\section{NOTES}

1. Ce travail est une version très remaniée d'une conférence présentée à la table ronde "Métamorphoses 2008 ", sous l'égide du PLH-CRATA, Université de Toulouse-Le Mirail, mai 2008.

2. Quelques exceptions notables figurent dans la revue Formules, 5, 2001, avec le centon mallarméen de Bernardo Schiavetta, sonnet intitulé Un éclat de ta voix (pp. 36-38), mais aussi les sonnets philosophiques de Michelle Grangaud, composés avec des séquences dodécasyllabiques toutes prélevées dans L'Être et le Néant de Jean-Paul Sartre (« Sartre poète », pp. 12-17).

3. C'était surtout le cas de l'Enéide qui, selon Armando Petrucci, apparaissait du $\mathrm{I}^{\mathrm{er}}$ au $\mathrm{VI}^{\mathrm{e}}$ siècle comme le repère absolu des lettrés romains (1981, p. 52).

4. Sur la manière subtile dont s'intègre à l'œuvre un fragment du récit virgilien, voir par exemple James D. Ellsworth (1988, pp. 47-55).

5. Sans jamais se borner à reprendre un schéma narratif prédisposé, Claudien s'appuie sur la double version ovidienne du mythe qu'il traite ( $M . V$, 341-661 et $F$. IV, 395-620), tout en multipliant les motifs et les réminiscences issus de Virgile. De manière symptomatique, l'amorce du livre 1 fait immédiatement écho à Ovide (rappel de M. V, 402-403 dans le v. 1), et presque aussitôt, à l'Enéide, avec l'évocation des Enfers (l'entame du v. 20 reprend En. VI, 264).

6. Comme le rappelle Giovanni Salanitro dans le panorama des centons antiques précédant son édition d'Hosidius Geta, une inscription (CIL 6271) signale un poeta Ouidianus, qui pourrait être un centoniste, à moins qu'il ne s'agisse d'un pasticheur (1981, p. 58).

7. Cet ensemble est conservé dans le Codex Salmasianus, qui remonte sans doute au viI ${ }^{\mathrm{e}}$ ou au VIII siècle (voir Scott McGill, 2005, p. xix ; édition d'Alexander Riese, Anthologia Latina I.1, Leipzig, 1894); les événements auxquels il se réfère conduisent à situer la compilation entre 523 et 534 . Cependant, parmi les centons que celle-ci comporte, seul l'Epithalame de Fridus, attribué à Luxorius, appartient avec certitude à cette période, sa composition ayant pu motiver le regroupement de poèmes analogues mais écrits antérieurement ; une telle hypothèse est d'autant plus admissible que la collection inclut la Médée d'Hosidius Geta, évoquée déjà par Tertullien comme un ouvrage récent dans les premières années du III ${ }^{\mathrm{e}}$ siècle (Traité de la prescription contre les hérétiques, XXXIX, 2-5).

8. Un troisième centon mythologique, consacré à l'histoire de Procné et Philomèle, correspond encore à une légende traitée par Ovide (M. VI, 412-674), mais adopte un scénario trop différent pour que l'on puisse envisager une filiation (voir Scott McGill, 2005, pp. 81-82). 
9. Comme le souligne Giovanni Salanitro, dans le volume où il réunit les centons anonymes du Codex Salmasianus qui n'avaient pas encore bénéficié d'une édition récente et soignée, cette pièce possède manifestement un « color Ovidianus » $(2009$, p. 27).

10. Une telle dimension n'a pas échappé à Scott McGill (2005, pp. 78-79), mais il n'a dégagé que les recoupements les plus notables; le repérage mérite donc d'être prolongé, afin de pouvoir en tirer toutes les leçons.

11. Une telle dichotomie s'applique lorsque le choix de fragments tirés d'un seul poète se conjugue avec l'adoption d'un modèle diégétique issu d'un autre écrit.

12. La lecture d'Ovide, telle qu'elle est attestée dans le centon, offre aussi un aperçu de la lecture qu'Ovide avait pu faire de Virgile.

13. - 1.33-35, dans la numérotation que fournit l'édition Garnier d'Ausone (1934). À trois reprises pourtant, le poète s'autorise, dans le Centon nuptial, une suite de deux vers (voir R. P. H. Green, 1991, p. 518).

14. Dans le Centon nuptial, on rencontre 26 vers isolés, soit plus d'un sur cinq.

15. Comme le montrent les relevés de David F. Bright (1984, p. 85).

16. Pour le texte du centon avec le repérage des fragments qui le composent, voir Annexe 1. Je reproduis, à de minimes variantes près dans la ponctuation, le texte établi par Giovanni Salanitro (2009, p. 28). Ma traduction s'attache à suivre d'aussi près que possible la structure des phrases et les effets de sens propres au texte latin.

17. Voir l'analyse de David F. Bright (1984, p. 85). Le centoniste a donc évité les segments d'un vers et demi, ainsi que le recours groupé, à cheval sur deux vers, d'hémistiches issus d'hexamètres consécutifs, modules qui, chez Ausone, interviennent respectivement 15 et 10 fois. Il a privilégié les extraits d'un demi vers, qui n'occupent chez Ausone qu'environ la moitié du poème.

18. Diffinduntur autem per caesuras omnes quam recipit uersus heroicus («d'autre part, on les découpe en recourant à toutes les césures qu'admet le vers héroïque ", 1. 35-36).

19. En effet, il s'amorce avec un segment qui correspond à un simple dactyle. Autrement, le centoniste s'appuie sur la coupe hephtémimère (v. 2, 5 et 8 ) et, de façon privilégiée, sur la penthémimère (v. 1, 3, 4, 10, 11, 13, 14, 15, 16).

20. L'anomalie est compensée par un solide lien entre les deux parties du vers, qui se rattachent à un contexte analogue dans l'œuvre-source, la contemplation par Enée à Carthage des peintures qui rappellent l'histoire de Troie (voir p. 88).

21. Rien ne prouve qu'ils aient été universellement connus et reconnus, en tant que normes, même si les observateurs modernes ont coutume de les envisager comme des principes communs à l'ensemble des centonistes latins.

22. Ausone émet ainsi le souhait «que des éléments trop serrés ne provoquent pas d'aspérités, que des éléments mal ajustés ne laissent pas entre eux de vide.» (densa ne supra modum protuberent, hiulca ne pateant, 1.57-58). Roberto Palla rapporte exclusivement ces consignes à la correction métrique des vers, ce qui semble en restreindre l'enjeu (1983, p. 288).

23. - 1.51-53, après avoir comparé l'exercice à celui d'un jeu, l'ostomachion, où il faut agencer des silhouettes diverses (objets, animaux, personnages) en combinant des tablettes triangulaires de tailles variées.

24. Je montrerai plus loin (pp. 84-85) combien le choix du fragment concerné dans l'œuvresource est justifié par les liens qu'il permet d'établir avec l'œuvre-cadre.

25. Une telle explication, que propose Scott McGill (2005, p. 77 n. 31), parait fort plausible, compte tenu du v. 13, qui valide après coup cet élément du décor. Le his renvoie sans nul doute à Narcisse et au jeune homme qu'il vient de découvrir (v. 8); mais il peut rappeler au surplus les trois noms communs du v. 9.

26. Ce besoin d'une harmonisation réciproque semble avoir été perçu très tôt, puisque Tertullien déjà le met en évidence, à propos notamment d'Hosidius Geta (De praescript. haer. XXXIX, 3) : « Tu 
vois qu'aujourd'hui l'on tire de Virgile une pièce absolument distincte, dont le sujet est agencé en fonction des vers disponibles, et les vers en fonction du sujet " (Vides hodie ex Virgilio fabulam in totum aliam componi, materia secundum uersus et uersibus secundum materiam concinnatis). Bien entendu, le participe concinnatis, qui signale le problème de l'ajustement, annonce l'exigence de concinnatio, d'harmonie, formulée cent cinquante ans plus tard par Ausone.

27. L'importance cruciale des inflexions sémantiques obtenues par les centonistes a justement été soulignée dans le travail de Rosa Lamacchia (1958, pp. 161 et 187) : la composition se devait de saisir les multiples virtualités syntaxiques et lexicales autorisant le cas échéant à employer certains extraits dans un contexte radicalement distinct. On vient d'en voir un exemple à propos du v. 10, entraînant un minime aménagement du texte virgilien.

28. 1979 , p. 107.

29. L'amusante étrangeté de tels effets a été soulignée par Scott McGill à propos d'Hosidius Geta, chez qui l'on finit par avoir l'impression que, dans plusieurs passages, Virgile a imité Sénèque (2002, p. 158).

30. Comme l'indique Giovanni Salanitro, « le centoniste manifeste là une exceptionnelle faculté de synthèse ! ( (« il centonario rivela qui rare doti di sintesi ! ", 2009, p. 27).

31. C'est Ovide, semble-t-il, qui avait imaginé de réunir les deux personnages (voir Gianpiero Rosati, 1983, p. 22). Ils figurent séparément dans les centons. Écho se rencontre dans le domaine grec, avec deux pièces anonymes: l'une se trouve dans l'Anthologie Palatine (IX, 382), l'autre, censée reproduire la prière de la nymphe poursuivie par le dieu Pan, est citée au VII ${ }^{\mathrm{e}}$ siècle par Héliodore, scholiaste du grammairien Denys le Thrace (Alfred Hilgard, 1979, p. 480). L'existence de ces compositions témoigne de l'intérêt spécial qu'offrent pour ce genre poétique des anecdotes évoquant un redoublement (voir Giovanni Polara, 1990, p. 274).

32. Sans compter les obstacles que le centoniste aurait dû affronter pour transcrire les rencontres de Narcisse avec une nymphe dont le nom n'apparait jamais chez Virgile, pas plus que le nom commun correspondant: on ne trouve que la locution équivalente en latin, uocis imago ( $G$. IV, 49).

33. Il faut admettre au préalable que, dans le centon, du fait de sa brièveté, une simple indication est susceptible de transposer tout un passage du texte ovidien.

34. C'est le nom candor qu'utilise le poète (la première occurrence étant assortie des qualificatifs eburneus et niueus). Mais on connaît le rôle particulier de l'adjectif candidus dans la poésie élégiaque, pour caractériser l'être aimé ; chez Ovide, notamment, il s'applique à Corinne (Am. I, 5, 10). Le terme initial du centon peut donc préfigurer en Narcisse le destinataire d'une passion que, pour le moment, il ne partage pas.

35. Chez Virgile, l'occurrence correspond à un groupe de jeunes gens qui s'exercent devant la ville du roi Latinus (En. VII, 162). Le centoniste impose donc une torsion à la valeur sémantique originelle de l'extrait. On pourrait parler à cet égard d'une annexion figurale: une synecdoque du nombre permet ici d'intégrer iuuentus et s'ajoute à celle de l'abstrait pour le concret, déjà présente dans l'Enéide.

36. Par contre, le centon ignore puer, bien plus fréquent chez Ovide (v. 352, 379, 413, 454, 495, 500).

37. Qu'il ait pour père un cours d'eau n'est pas repris dans le centon. Mais ce détail pourrait en quelque sorte resurgir au v. 13, sous les espèces du cours d'eau où se mire Narcisse, au lieu de la source ovidienne.

38. En somme, sans transition, le mode narratif passe de la focalisation interne à la focalisation zéro (sur ces catégories, voir Gérard Genette, 1972, pp. 206-224). Certes, dans son principe, la métalepse narrative, telle que la conçoit Gérard Genette, se rapporte à la voix du récit, non à son mode, puisqu'elle est définie comme une transgression du niveau d'enchâssement : par exemple quand un narrateur hétérodiégétique s'adresse aux personnages (2004, p. 14). Mais on peut établir par extension un type de figure où c'est le régime modal de la narration qui est transgressé. 
39. Ainsi le v. 425 : Se cupit imprudens et qui probat ipse probatur. ("Sans le comprendre, il se désire lui-même et celui qui admire est aussi admiré »).

40. Le centoniste détourne un extrait de l'Enéide où Pygmalion, le frère de Didon, assassine Sychée, l'époux de sa sœur, sans redouter l'amour de cette dernière pour son mari (securus amorum, En. I, 350). On retrouve un fragment du même passage dans le second hémistiche du v. 14.

41. La correspondance entre les deux fins de vers est d'autant plus intéressante qu'elle rapproche des hexamètres virgiliens où il est question des passions conflictuelles éprouvées par Didon, pour Enée d'une part, quand, sous l'emprise de Cupidon, elle s'éprend du Troyen (En. I, 749), pour Sychée d'autre part, dont le souvenir la tourmentera par la suite (En. I, 350).

42. Comme le centon laisse de côté la rencontre avec la nymphe Écho, il est normal qu'une telle évocation advienne dès ses premiers vers, se conjuguant avec la présentation de Narcisse.

43. Chez Ovide, les trois éléments constitutifs sont repris au moment où Narcisse tombe amoureux de son image : v. 427-429 pour la source, v. 437 pour l'herbe et v. 441 pour les bois.

44. Le déictique haec paraît accompagner ces termes, comme s'ils rappelaient des indications; mais puisque rien de tel ne précède, il ne saurait jouer le rôle d'adjectif démonstratif ; il est plus probable d'y voir un pronom, comme dans l'extrait de Virgile, en B. II, 4 : au lieu d'un accusatif neutre pluriel, on a un nominatif féminin singulier qui reprend iuuentus.

45. Dans l'extrait virgilien ( $G$. IV, 1), elle s'applique au miel, dont il va être question dans tout le livre IV des Géorgiques. On remarque alors que la mention de la source, au v. 3 du centon, qui est coordonnée avec cette fin du v. 2, vient d'un passage, dans la première Bucolique (v. 52), où le repos dans la fraîcheur d'une campagne humide est couplé avec le murmure des abeilles parmi les fleurs (v. 54). De la sorte, apparaît un principe d'analogie contextuelle, qui favorise l'assemblage de fragments, dénués en eux-mêmes de similitudes, mais liés par leur alentour immédiat dans l'œuvre-source. Pour un phénomène du même ordre, où, cette fois, le voisinage d'une formule identique provoque le rapprochement, voir Scott McGill (2005, p. 77) : il s'agit des deux extraits qui forment le v. 8. En ce qui concerne les v. 2 et 3, il n'est pas absurde d'estimer que la possible association d'idées entre le site et le travail des abeilles, est apte à évoquer par métonymie, pour des lecteurs imbus des œuvres virgiliennes, quelque espace verdoyant et fleuri.

46. Peu auparavant, dans le même livre des Métamorphoses, c'est aussi d'une transgression involontaire qu'Actéon se rend coupable, en un site analogue (v. 155-162). Perrine Galand-Hallyn a montré comment y sont distribués au cours de la description les indices cachés d'une fatalité menaçant le héros (1994, p. 224). Plus généralement, Gianna Petrone souligne l'ambivalence d'un tel type de paysage, susceptible de représenter un ordre naturel pacifié, mais aussi d'accueillir le déchaînement d'une sauvagerie latente (1998, pp. 177-195).

47. Dumque sitim sedare cupit, sitis altera creuit ("Alors qu'il voulait étancher sa soif, une soif nouvelle a surgi en lui ", v. 415). Cette similitude est commentée par Scott McGill (2005, p. 79).

48. La rareté de cette image chez Virgile est soulignée par Jacques Perret dans son édition de l' Enéide; la formule y concerne une passion qui sera fatale à Didon, comme dans le centon l'amour va perdre le héros.

49. Un début d'hexamètre s'offrait ainsi, plus proche de l'œuvre-cadre, mais dépourvu de l'image commune : et bibit humorem (G. II, 218 : il s'agit d'une terre qui boit l'humidité). Il existait aussi un équivalent sémantique, hausit, par exemple dans En. I, 738 ou IX, 23.

50. C'est fons qu'Ovide utilise le plus souvent; au v. 476, il remplace le terme par lacus.

51. Il est vrai que flumen désigne parfois des eaux stagnantes, comme chez Horace $S .1,1,69$, à propos du supplice de Tantale, passage repris dans Properce 2, 17, 5 (je dois ces références au réviseur de la revue Dictynna); pourtant, l'absence du terme dans le développement des Métamorphoses ne le prédisposait guère à fournir un équivalent de fons.

52. En ce cas, deux éléments liés dans l'œuvre-source influent sur le centon, même si un seul d'entre eux s'y trouve effectivement inclus. On reconnaît, à propos du même passage, le principe 
d'analogie contextuelle évoqué plus haut (n. 45). Du reste, les ressemblances entre le v. 2 du centon et B. I, 51 ne manquent pas de frapper un observateur attentif : on retrouve un adverbe de lieu, un nom suivi d'un adjectif au neutre pluriel, comme si les choix du centoniste se réglaient sur des corrélations dont l'ampleur outrepasse celle des fragments retenus. Davantage, le second extrait du v. 13 se trouve surdéterminé par une corrélation avec le segment initial du centon: celui-ci marque, dans l'Enéide, l'apparition de la truie blanche qui présage la fondation d'Albe (En. VIII, 82) ; celui-là correspond, dans les prophéties d'Hélénus, à l'annonce même de ce présage (En. III, 389). Un tel constat donne à percevoir la densité des liens entre bien des fragments.

53. Voir p. 74.

54. Une indication métaphorique, v.414, prédisposait une telle approche: Narcisse vient s'étendre, "séduit par la figure du site", faciem... loci... secutus. L'emploi de facies tend à personnifier le décor, annonçant du même coup le visage que le héros découvrira dans l'onde (ce terme, à l'ablatif dans le centon, v. 4, désigne justement la figure de Narcisse). On sait d'ailleurs combien, dans l'histoire de Céphale et Procris, Ovide a su jouer sur une ambivalence analogue : les remerciements que le héros adresse à la brise bienfaisante peuvent laisser croire qu'il parle à une amante (M. VII, 812-823). Au v. 3 du centon, les louanges de Narcisse pour le lieu semblent répondre de manière fugitive à cette scène.

55. En retour, les «divins agréments » du paysage, caelestia dona (v. 2), peuvent faire songer à l'insistance des Métamorphoses sur la splendeur de l'adolescent, telle que lui-même la découvre dans le miroir des eaux (v. 420-421). Ses yeux sont assimilés à des astres et sa chevelure comparée à celle de Bacchus ou d'Apollon.

56. Dans les Métamorphoses, comme l'épisode illustre la science prophétique de Tirésias, le dénouement est annoncé dès le début (v. 348-350).

57. L'hexamètre chez Virgile, correspond aux paroles d'Andromaque retrouvant chez Ascagne l'apparence d'Astyanax, son fils perdu.

58. Ainsi qu'à $M$. III, 473, qui joue sur la coïncidence entre amour et mourir pour les deux amants qui ne font qu'un (union à laquelle répond bien celle des deux combattants troyens). Pour un relevé complet des échos variés dans le centon, provenant de tout l'épisode ovidien, voir l'Annexe 2.

59. Voir p. 78.

60. Le v. 9, tout en laissant subsister une incertitude, amorce la révélation en soulignant la ressemblance exceptionnelle des deux adolescents.

61. Une possible influence des représentations plastiques, ramenant à la simple image de Narcisse penché vers son reflet, ne saurait jouer exclusivement, l'usage de l'imparfait jusqu'à la fin attestant la dimension narrative du poème.

62. L'extrait virgilien est un hexamètre décrivant Enée lorsqu'il regarde sans comprendre leur sens les scènes ciselées par Vulcain sur son bouclier (En. VIII, 730); déjà les fragments du v. 6 provenaient de la scène où le chef troyen parcourt, à Carthage, les scènes qui représentent sur un temple la guerre de Troie $(E n . ~ I, 495+464)$. Ainsi le centoniste retient dans l'œuvre-source des composantes liées au spectacle d'images. Bien plus, comme l'a montré Scott McGill, le choix de plusieurs morceaux, dans la seconde moitié du centon, semble surdéterminé par leur proximité avec l'évocation, dans leur contexte originel, d'une imago qui correspond soit à une ressemblance frappante, soit à l'apparition d'un spectre (2005, p. 77 : v. 9, cf. En. III, 489 ; v. 14, cf. En. I, 353-354 ; v. 16, cf. En. II, 793 et VI, 701). En outre, le second extrait du v. 11 provient d'un passage où Enée voit en rêve l'image de Mercure, identique au dieu lui-même (forma sert alors à désigner cette apparence, En. IV, 556).

63. Il provient des deux vers identiques, dont le second hémistiche a fourni par ailleurs l'extrait terminal du v. 15 (En. II, 792ou VI, 700).

64. Chez Virgile, il s'agit de Dédale, qui n'a pas réussi à représenter la mort de son fils Icare (En. VI, 32). 
65. Il n'existe aucun autre morceau analogue dans l'Enéide. Comme le souligne Scott McGill, une équivalence est établie entre ces personnages fantomatiques et l'être illusoire que désire Narcisse (2005, p. 30).

66. Celui-ci en latin est conçu comme une modalité du reflet, imago uocis désignant l'écho (comme au v. 385).

67. Par la même occasion, l'ordre entre collum et bracchia retrouve celui du modèle virgilien. Dans l'extrait suivant du centon, le nec initial renforce la correspondance.

68. Les termes communs à Ovide et au centon figurent en caractères gras. Interverties, les deux portions de l'hexamètre virgilien sont disposées à la charnière des v. 15 et 16 .

69. Il n'est pas indispensable de postuler que la recherche d'une telle implication ait guidé le choix du centoniste : l'écriture a pu bénéficier d'une virtualité propre aux matériaux mobilisés chez Virgile, rappelant à la fois les deux passages associés dans les Métamorphoses.

70. Si l'adjectif marquant la couleur diffère, albus se retrouve lui aussi, juste à la fin de l'hexamètre virgilien (En. VIII, 82) dont le début est inséré v. 1. On note, accentuant la similitude entre les passages, une paronomase entre inueniunt et ueniebat.

71. Cette circularité, virtuelle en quelque sorte, puisqu'il n'y a pas de correspondance terme à terme entre le début et la fin du centon, se trouve relayée, dans le rapport à l'œuvre-source, par une corrélation métonymique, renforçant le dispositif. En effet, un lien spécial associe deux fragments presque symétriques, le début du v. 2 et la fin du v. 16 : ils appartiennent chacun à l'introduction de la seconde Bucolique, dont le thème est l'échec programmé d'un amour, celui de Corydon pour Alexis. De la sorte, l'ultime segment du poème suggère un retour vers le point de départ.

72. On peut se demander si le caractère insolite d'une telle formule, en retenant l'attention sur le vers, n'a pas valeur d'indice.

73. Maddalena Vallozza dénonce de la sorte son «obscurité ainsi qu'une mise en œuvre insignifiante des règles de composition propres aux centons » $(1986$, p. 336).

74. Giovanni Salanitro incite à la prudence en signalant que la comparaison avec Ovide risque de faire tort au poème (1997, p. 2339). D'ailleurs, il le caractérise lui-même comme l'un des plus réussis parmi les centons anonymes du Codex Salmasianus (2009, p. 27).

75. 2005, pp. 77-79.

76. Voir ci-dessus, p. 79.

77. Les deux syllabes communes occupent la même place dans l'hexamètre. Ce recoupement intervient deux vers après celui de bibit, installant une convergence des similitudes; divers autres échos se disposent sur la presque totalité du centon: l'Annexe 3 en présente une vue d'ensemble.

78. On découvre à nouveau dumque à cette place dans les v. 426 et 480 . Corrélativement, l'emploi de dum se répète dans le centon (v. 10). Quant au segment dum stupet, au v. 6, il se détache d'autant mieux qu'il forme un extrait dont la découpe dérogatoire est unique dans le poème, on l'a signalé p. 5 .

79. Le utque de ce vers trouve un corrélat paronomastique dans le atque du centon, amorçant le second extrait du v. 6.

80. On peut rapprocher le se quoque... agnouit virgilien, "lui aussi, il s'est reconnu», et la prophétie de Tirésias à propos de Narcisse : il vivra longtemps «s'il ne se connaît pas » (si se non nouerit, M. III, 348).

81. Le v. 513 s'amorce par obstipuit, composé de stupet. Ovide a repris l'agencement, dont il a juste espacé un peu plus les occurrences tout en accentuant leur similitude, adstupet faisant suite à stupet.

82. Les caractères gras indiquent les corrélations verbales entre Ovide et Virgile, leur soulignement ce qui en réapparaît dans le centon. Complétant le rapport du v. 6 avec les deux passages d'Ovide, le début du v. 5 correspond sur le plan thématique à la fin de M. III, 381 . 
83. Le terme, chez Virgile, s'applique à Evandre, qui fait ses adieux à son fils Pallas (En. VIII, 559). La corrélation entre le centon et l'hexamètre ovidien reçoit plusieurs appoints : la syllabe initiale de mentem prolonge la ressemblance verbale, en répondant à celle de mendacem; le nom formam à la fin de M. III, 439, figure au nominatif dans le v. 8 du centon.

84. Même si les termes diffèrent, avec le gérondif tuendo issu de Virgile en contrepartie du substantif lumine chez Ovide. Quant au verbe ardescit, il se relie à la forme simple ardet (M. III, 426), celle-ci figurant par ailleurs dans l'Enéide à propos de la passion dévorante qu'éprouve la reine (IV, 101). L'inchoatif peut même être mis en relation avec un passage ovidien qui se rapporte à la vive passion de la nymphe Écho : on trouve alors calescit (M. III, 372).

85. Une autre occurrence de cette forme, alliée à spem, se trouve dans les paroles de Narcisse, au v. 457.

86. Il est courant de trouver chez Virgile amantem en fin de vers (G. IV, 488; En. I, 352 ; IV, 296, 479 , outre amantem est, v. 370) : le centoniste, ayant discerné sa reprise chez Ovide, a mis à profit la similitude.

87. Scott McGill relève cette corrélation en signalant également la connexion entre l'adjectif decora au v. 11 et decus, « le charme » qui, dans le récit d'Ovide, v. 422, sert de complément d'objet à miratur (2005, p. 79).

88. Le centoniste mobilise ainsi des extraits renvoyant à un ensemble de scènes analogiquement liées, où le regard d'Enée parcourt des œuvres plastiques dans des circonstances décisives pour lui : v. 6, représentation de la guerre de Troie, sur le temple de Carthage, préfigurant les récits du héros; v. 12, annonce d'un futur victorieux, sur les armes amenées par Vénus; v. 16, portes ouvrées par Dédale pour le temple d'Apollon, préludant à l'initiatique descente aux Enfers.

89. Ces échos se fondent sur quelques mots, souvent liés les uns aux autres, non sur des syntagmes analogues, comme il arrive souvent dans la poésie latine, quand la récriture n'est pas tributaire des contraintes inhérentes au centon.

90. L'Annexe 3 les fait ressortir suffisamment pour qu'il ne soit pas indispensable d'en commenter ici tout le détail. En outre, on trouve dans l'Annexe 2 les données relatives à d'autres passages.

91. Je l'ai montré, p. 86.

92. De même que pour stupet (v. 6), comme on l'a vu, pp. 88-89.

93. Cet ablatif ainsi placé dans l'hexamètre est récurrent dans l'Enéide: outre l'extrait qui correspond à VIII, 730, on le rencontre en IV, 84 et VI, 293, dont la fin a inspiré celle de M. III, 416. 94. Gianpiero Rosati a bien mis en évidence une telle dimension (1983, p. 26 notamment). Il souligne aussi la manière dont le thème de l'illusion développé dans l'anecdote débouche sur une mise en scène de l'illusion littéraire (ibid., pp. 44-46).

95. On sait que Virgile l'a trouvée chez Apollonios de Rhodes, avec la passion qu'Eros allume pour Jason dans le cœur de Médée (Arg. III, 286-297).

96. Le v. 464 dans le discours de Narcisse ne fait que réitérer ces occurrences.

97. Les trois occurrences recoupent les formulations de l'Enéide, au début du livre IV : ardet (M. III, 426/En. IV, 101), uritur (M. III, 430/En. IV, 68), et une même fin d'hexamètre, carpitur igni (M. III, $490 / E n$. IV , 2). Ces corrélations ne sont pas intégrées au centon, mais offrent comme un arrièreplan métonymique des extraits retenus qui présentent la même image.

98. L'Annexe 2 permet d'observer la spécificité des correspondances.

99. Une image identique, dans l'Enéide, s'applique à Didon : est mollis flamma medullas (« la flamme dévore ses tendres moelles", IV, 66). Mais le centoniste a préféré à ce fragment, qui d'ailleurs occupe la seconde moitié d'un hexamètre, un extrait lié à l'incendie de Troie (En. II, 758) et intégré par le détour d'une métaphorisation: c'est qu'il comporte le vocable ignis, commun avec M. III, 490.

100. Il meurt, on le sait, de ne plus manger, parce qu'un autre appétit le ronge (M. III, 437-438); puis son corps s'exténue, parce qu'il sert d'aliment à une ardeur incontrôlée (v. 479). 
101. Narcisse lui-même souligne le paradoxe de sa condition : la proximité maximale de l'objet aimé détermine pour lui sa totale inaccessibilité (M. III, 453).

102. Scott McGill n'a pas manqué de relever cette figure (2005, p. 78). En outre, dans un contexte où pascit active l'image de la nourriture, inanis, par association d'idées, suggère l'inanition, expleri renvoyant de son côté au rassasiement. La métaphore filée gagne ainsi une occurrence et le rapport oxymorique se redouble.

103. Scott McGill souligne le retour de cette alliance à valeur d'oxymore dans deux autres poèmes de l'Anthologie latine qui traitent également de Narcisse (2005, p. 78). Mais le motif, dans le centon, procède avant tout du récit ovidien, qui rapproche notamment ardet et fonti à la fin de vers successifs (M. III, 426-427).

104. Certes, dans le centon, ignis n'est qu'à la seconde place, mais il voisine avec l'adverbe ilicet, apte à rappeler, selon une paronomase, les eaux que dénote à la même place la forme liquitur en M. III, 490. En outre, un double écho implicite se trame avec la prophétie d'Hélénus, dont provient le second extrait du vers 13 (En. III, 389) : le devin annonce l'apparition de la truie blanche, aux yeux d'Enée « inquiet », sollicito (dans le premier hémistiche), sous des « chênes verts » (ilicibus, v. 390). De nouveau, le principe d'analogie contextuelle tisse des rapports avec les extraits intégrés par le centoniste.

105. Un regard aiguisé par l'observation peut dès lors percevoir une structure dérivée, propre au centon qui se relie à la métaphore du feu : la proximité corrélative d'une série paronomastique fondée sur ignis; d'une part, précédant le verbe ardescit au v. 6, les deux formes homologues insigni puis insignis (v. 3 et 4); de l'autre, l'adjectif ignarus au v. 12, qui semble annoncer l'occurrence du v. 13.

106. Une telle dimension n'est pas réservée au centon de Narcisse : ainsi la Médée d'Hosidius Geta apparaît-elle comme tributaire de Sénèque, mais aussi d'Ovide, dans la douzième Héroïde notamment (Giovanni Salanitro, 1997, p. 2338); ainsi l'Epithalamium Fridi, de Luxorius, est-il redevable à Stace en particulier (Zoja Pavlovskis, 1965 : p. 173).

107. « la struttura esteriore stessa dei centoni porta a sentire l'azione cantata in rapporto di stretta subordinazzione a tutti quegli elementi costruttivi che in qualche maniera comportano l'intervento della riflessione " (1980, p. 23).

108. Elle assimile un tel effort au souci de clarté des écrivains participant à l'Oulipo lorsqu'ils explicitent le programme qui régit l'écriture de telle ou telle œuvre à contrainte $(1979$, p. 89).

109. La condamnation que formule Dumarsais contre le principe même de la contrainte, c'est le terme qu'il emploie, est à cet égard révélatrice : «il vaut mieux s'occuper à bien penser, à bien exprimer ce qu'on pense, qu'à perdre le temps à un travail où l'esprit est toujours dans les entraves, où la pensée est subordonnée aux mots » (1988, p. 219).

110. On peut alors considérer qu'un tel outrepassement des exigences représentatives par le dispositif qui la permet correspond à un régime particulier de l'écrit, poétique notamment, dont une discipline nouvelle, la textique, fournit le concept sous le vocable, de métareprésentation (pour une mise en œuvre d'un tel concept à propos de la poésie ovidienne, voir Gilles Tronchet, 2007, pp. 172 et 178).

111. J'ai proposé de la nommer fiction archétypale (Gilles Tronchet, 1998, p. 34).

112. Au v. 10 du centon, le pendit, issu d'un pendet virgilien (voir p. 6) condense en quelque sorte deux verbes de M. III, 426, petit et accendit. 


\section{RÉSUMÉS}

Le centon anonyme de Narcisse offre un exemple marquant de la virtuosité avec laquelle l'écriture, dans ce genre poétique, s'adapte souvent à une double exigence : l'usage exclusif de fragments tirés de Virgile (on peut parler d'œuvre-source) et l'adoption d'un modèle narratif tout autre, ici l'épisode ovidien des Métamorphoses (on peut parler d'œuvre-cadre). Par un choix savant d'extraits, le centoniste est parvenu non seulement à restituer maints aspects de la diégèse ovidienne, mais aussi à introduire, grâce au matériau virgilien, nombre d'échos à l'œuvre d'Ovide, comme si paradoxalement Virgile s'était inspiré des vers de son successeur. Ce double jeu, que l'on retrouve notamment dans la Médée d'Hosidius Geta, manifeste, dans la composition des centons profanes antiques, un raffinement qui méritait d'être exploré.

INDEX

Mots-clés : centon, écriture à contrainte, intertextualité, Métamorphoses, métapoétique

\section{AUTEUR}

\section{GILLES TRONCHET}

Université de Nantes

giltr@wanadoo.fr 